\title{
ENHANCED SPECIALIZATION AND MICROLOCALIZATION
}

\author{
ANDREA D'AGNOLO AND MASAKI KASHIWARA
}

\begin{abstract}
Enhanced ind-sheaves provide a suitable framework for the irregular Riemann-Hilbert correspondence. In this paper, we show how Sato's specialization and microlocalization functors have a natural enhancement, and discuss some of their properties.
\end{abstract}

\section{Contents}

1. Introduction 1

2. Bordered normal deformation 3

3. Review on enhanced ind-sheaves 8

4. Specialization 9

5. Fourier-Sato transform and microlocalization 19

6. Specialization at $\infty$ on vector bundles 24

References $\quad 28$

\section{INTRODUCTION}

1.1. Let $M$ be a real analytic manifold, and $N \subset M$ a closed submanifold. The normal deformation (or deformation to the normal cone) of $M$ along $N$ is a real analytic manifold $M_{N}^{\text {nd }}$ endowed with a map $(p, s): M_{N}^{\text {nd }} \rightarrow M \times \mathbb{R}$, such that $s^{-1}\left(\mathbb{R}_{\neq 0}\right) \stackrel{\sim}{\sim} M \times \mathbb{R}_{\neq 0}$ and $s^{-1}(0)$ is identified with the normal bundle $T_{N} M$.

Sato's specialization functor $\nu_{N}$, defined through $p: M_{N}^{\text {nd }} \rightarrow M$, associates to a sheaf ${ }^{1} F \in \mathrm{D}^{\mathrm{b}}\left(\mathbf{k}_{M}\right)$ a conic sheaf on $T_{N} M$, describing the asymptotic behaviour of $F$ along $N$. Let $\dot{T}_{N} M$ be the complement of the

2010 Mathematics Subject Classification. Primary 32C38, 35A27, 14 F05.

Key words and phrases. Sato's specialization and microlocalization, Fourier-Sato transform, irregular Riemann-Hilbert correspondence, enhanced perverse sheaves.

The research of A.D'A. was partially supported by GNAMPA/INdAM. He acknowledges the kind hospitality at RIMS of Kyoto University during the preparation of this paper.

The research of M.K. was supported by Grant-in-Aid for Scientific Research (B) 15H03608, Japan Society for the Promotion of Science.

${ }^{1}$ We abusively call sheaf an object of the bounded derived category $\mathrm{D}^{\mathrm{b}}\left(\mathbf{k}_{M}\right)$ of sheaves of $\mathbf{k}$-vector spaces on $M$, for a fixed base field $\mathbf{k}$. 
zero section, identified with $N$. One has $\left.\left.\nu_{N}(F)\right|_{N} \simeq F\right|_{N}$, and $\left.\nu_{N}(F)\right|_{\dot{T}_{N} M}$ only depends on $\left.F\right|_{M \backslash N}$.

Sato's microlocalization functor $\mu_{N}$ is obtained from $\nu_{N}$ by FourierSato transform, and provides a tool for the microlocal analysis of $F$ on the conormal bundle $T_{N}^{*} M$.

1.2. In this paper, we will define the enhanced version of the specialization and microlocalization functors. With notations as in $\S 1.1$, this proceeds as follows.

We start by showing that there exists a (unique) real analytic bordered space $\left(M_{N}^{\text {nd }}\right)_{\infty}$ such that the map $p:\left(M_{N}^{\text {nd }}\right)_{\infty} \rightarrow M$ is semiproper. (We call this a bordered compactification of $p$.)

Mimicking the classical construction, with $M_{N}^{\text {nd }}$ replaced by $\left(M_{N}^{\text {nd }}\right)_{\infty}$, we get an enhancement of Sato's specialization. This associates to an enhanced ind-sheaf $K \in \mathrm{E}^{\mathrm{b}}\left(\mathrm{Ik}_{M}\right)$ a conic enhanced ind-sheaf $\mathrm{E} \nu_{N}(K)$ on the bordered compactification $\left(T_{N} M\right)_{\infty}$ of $T_{N} M \rightarrow N$. Consider the bordered space $(M \backslash N)_{\infty}:=(M \backslash N, M)$. One has $\left.\left.\mathrm{E} \nu_{N}(K)\right|_{N} \simeq K\right|_{N}$, and $\left.\mathrm{E} \nu_{N}(K)\right|_{\left(\dot{T}_{N} M\right)_{\infty}}$ depends on $\left.K\right|_{(M \backslash N)_{\infty}}$, not only on $\left.K\right|_{M \backslash N}$.

Then, using the enhanced Fourier-Sato transform ${ }^{L}(\cdot)$, we get the enhanced microlocalization functor $\mathrm{E} \mu_{N}:={ }^{\mathrm{L}} \mathrm{E} \nu_{N}$, with values in conic enhanced ind-sheaves on $\left(T_{N}^{*} M\right)_{\infty}$.

We establish some functorial properties of the functors $\mathrm{E} \nu_{N}$ and $\mathrm{E} \mu_{N}$. These are for the most part analogous to properties of the classical functors $\nu_{N}$ and $\mu_{N}$, but often require more geometrical proofs.

1.3. In view of future applications to the Fourier-Laplace transform of holonomic $\mathcal{D}$-modules, we also discuss the following situation.

Let $\tau: V \rightarrow N$ be a vector bundle, and $V_{\infty}$ its bordered compactification. In this setting, we consider the natural enhancement of the smash functor from $[2, \S 6.1]$, described as follows. Let $\mathbb{S} V:=((\mathbb{R} \times V) \backslash$ $(\{0\} \times N)) / \mathbb{R}_{>0}^{\times}$be the fiberwise sphere compactification of $\tau$, and identify $V$ with the hemisphere $\left(\mathbb{R}_{>0} \times V\right) / \mathbb{R}_{>0}^{\times}$. Consider the hypersurface $H:=\partial V \subset \mathbb{S} V$, and identify $\dot{V}:=V \backslash N$ with the half of $T_{H}(\mathbb{S} V)$ pointing to $V$. Then, the restriction of $\mathrm{E} \nu_{H}$ to $(\dot{V})_{\infty}$ can be thought of as a "specialization at infinity" on $V$. The enhanced smash functor $\mathrm{E} \sigma_{V}$ (see $\S 6.1)$ provides an extension of $\mathrm{E} \nu_{H}$ from $(\dot{V})_{\infty}$ to $V_{\infty}$.

If $K$ is an enhanced ind-sheaf on $V_{\infty}$, with the natural identification $V_{\infty}^{*} \simeq\left(T_{N}^{*} V\right)_{\infty}$ one has (see Proposition 6.6)

$$
\mathrm{E} \sigma_{V^{*}}\left({ }^{\mathrm{L}} K\right) \simeq \mathrm{E} \mu_{N}(K) .
$$

1.4. The contents of this paper are as follows.

We introduce in Section 2 the notion of bordered compactification. This is a relative analogue of the classical one-point compactification. 
Then, we show that the normal deformation $p: M_{N}^{\text {nd }} \rightarrow M$ has a bordered compactification $\left(M_{N}^{\text {nd }}\right)_{\infty}$ in the category of subanalytic bordered spaces.

Using the bordered normal deformation, and after recalling some notations in Section 3, the enhanced specialization is introduced and studied in Section 4. We also consider an analogous construction, attached to the real oriented blow-up of $M$ with center $N$. Moreover, we discuss the notion of conic enhanced ind-sheaf.

Section 5 establishes some complementary results on the enhanced Fourier-Sato transform, and uses it to enhance the microlocalization functor. Finally, in Section 6, we link the microlocalization along the zero section of a vector bundle with the so-called smash functor.

\section{BORDERED NORMAL DEFORMATION}

Here, after recalling the notion of bordered space from [3, §3], we introduce the notion of bordered compactification. We then show that the deformation to the normal cone, for which we refer to [7, 44.1$]$, admits a canonical bordered compactification.

In this paper, a good space is a topological space which is Hausdorff, locally compact, countable at infinity, and with finite soft dimension.

2.1. Bordered spaces. Denote by $\mathcal{T}$ op the category of good spaces and continuous maps.

Denote by $b$ - $\mathcal{T}_{\text {op }}$ the category of bordered spaces, whose objects are pairs $\mathrm{M}=(M, C)$ with $M$ an open subset of a good space $C$. Set $\stackrel{\circ}{\mathrm{M}}:=M$

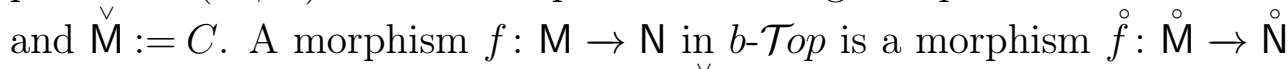

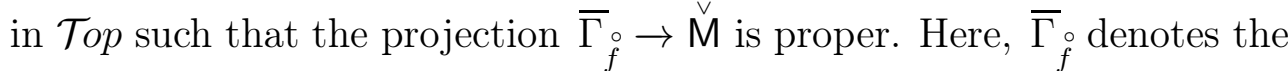
closure in $\stackrel{\vee}{\mathrm{M}} \times \stackrel{\mathrm{N}}{\mathrm{N}}$ of the graph $\Gamma_{f}$ of $\stackrel{\circ}{f}$.

The functor $\mathrm{M} \mapsto \stackrel{\circ}{\mathrm{M}}$ is right adjoint to the embedding $\mathcal{T}$ op $\rightarrow b$ - $\mathcal{T}$ op, $M \mapsto(M, M)$. We will write for short $M=(M, M)$. Note that $\mathrm{M} \mapsto \mathrm{M}$ is not a functor.

We say that $f: \mathrm{M} \rightarrow \mathrm{N}$ is semiproper if $\bar{\Gamma}_{f} \rightarrow \mathrm{N}$ is proper. We say that $M$ is semiproper if so is the natural morphism $M \rightarrow\{p t\}$. We say

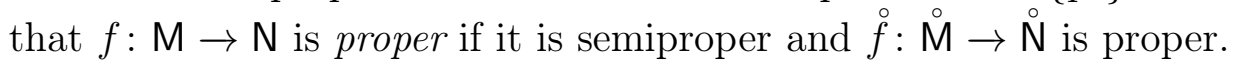

For any bordered space $M$ there are canonical morphisms

$$
\stackrel{\circ}{\stackrel{i_{\mathrm{M}}}{\longrightarrow}} \mathrm{M} \stackrel{j_{\mathrm{M}}}{\longrightarrow} \stackrel{\vee}{\mathrm{M}}
$$

Note that $j_{\mathrm{M}}$ is semiproper.

By definition, a subset $Z$ of $\mathrm{M}$ is a subset of $\stackrel{\circ}{\mathrm{M}}$. We say that $Z$ is open (resp. locally closed) if it is so in $\stackrel{\circ}{\mathrm{M}}$. For a locally closed subset $Z$ of $\mathrm{M}$, we set $Z_{\infty}=(Z, \bar{Z})$ where $\bar{Z}$ is the closure of $Z$ in $\stackrel{\vee}{ }$. Note that, for an

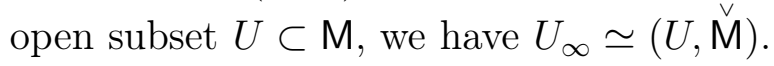


We say that $Z$ is relatively compact in $\mathrm{M}$ if it is contained in a compact subset of $\mathbf{M}$. Then, for a morphism of bordered spaces $f: M \rightarrow N$, the image $f(Z)$ is relatively compact in $\mathrm{N}$. In particular, the condition of $Z$ being relatively compact in $M$ does not depend on the choice of $M$.

2.2. Bordered compactification. Let $\mathrm{S}$ be a bordered space. Denote by $b$ - $\mathcal{T}_{o p_{\mathrm{S}}}$ the category of bordered spaces over $\mathrm{S}$, and by $\mathcal{T}_{o p_{\mathrm{S}}}$ the category of good spaces over $\stackrel{\text { S}}{\text {. }}$

Lemma 2.1. Let $\mathrm{M}$ and $\mathrm{N}$ be bordered spaces over $\mathrm{S}$. If $\mathrm{N}$ is semiproper over $\mathrm{S}$, then the natural morphism

$$
\operatorname{Hom}_{b-\mathcal{T}_{o p} \mathrm{~s}}(\mathrm{M}, \mathrm{N}) \rightarrow \operatorname{Hom}_{\mathcal{T}_{o p_{\mathrm{S}}}}(\stackrel{\circ}{\mathrm{M}}, \stackrel{\circ}{\mathrm{N}})
$$

is an isomorphism.

Proof. Denote by $p: \mathrm{M} \rightarrow \mathrm{S}$ and $q: \mathrm{N} \rightarrow \mathrm{S}$ the given morphisms. In order to prove the statement, it is enough to show that any continuous map $\stackrel{\circ}{f} \stackrel{\circ}{\mathrm{M}} \rightarrow \stackrel{\circ}{\mathrm{N}}$ which enters the commutative diagram

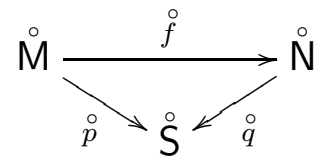

induces a morphism $f: \mathrm{M} \rightarrow \mathrm{N}$. That is, we have to prove that the map $\bar{\Gamma}_{f} \rightarrow \stackrel{v}{ }$ is proper.

It is not restrictive to assume that $\stackrel{\circ}{p}$ extends to a map $\stackrel{\vee}{M} \rightarrow \stackrel{v}{S}$. Since

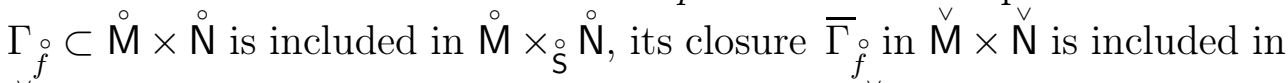
$\stackrel{v}{M} \times_{v} \bar{\Gamma}_{q}$. Since $q$ is semiproper, the map $\bar{\Gamma}_{q} \rightarrow \stackrel{\mathrm{S}}{ }$ is proper. Hence so is the map $\bar{\Gamma}_{\circ} \rightarrow \stackrel{\text { M. }}{ }$.

Proposition 2.2. Let $M$ be a good space, and $p: M \rightarrow \mathrm{S}$ a morphism of bordered spaces. Then there exists a bordered space $M_{\infty}$, with $\left(M_{\infty}\right)^{\circ}=$ $M$, such that $\stackrel{\circ}{p}$ induces a semiproper morphism $p_{\infty}: M_{\infty} \rightarrow$ S. Such an $M_{\infty}$ is unique up to a unique isomorphism.

Definition 2.3. With notations as above, $M_{\infty}$ is called the bordered compactification of $M$ over S.

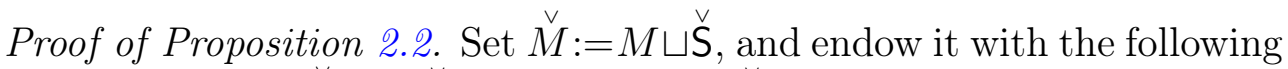

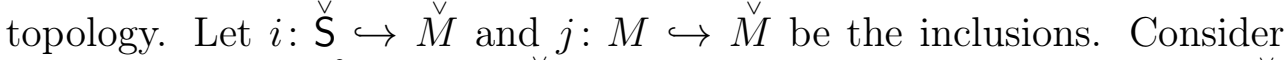
the map $\stackrel{\circ}{p}: M \rightarrow \stackrel{\circ}{\mathrm{S}}$. For $s \in \stackrel{\mathrm{S}}{\mathrm{S}}$, a neighborhood of $i(s)$ is a subset of $\stackrel{\vee}{M}$ containing $i(V) \cup j\left(\stackrel{\circ}{p}^{-1}(V \cap \stackrel{\circ}{S}) \backslash K\right)$, where $V \subset \stackrel{\vee}{S}$ is a neighborhood of $s$, and $K \subset M$ is a compact subset. For $x \in M$, a neighborhood of $j(x)$ is a subset of $M$ containing $j(U)$, where $U \subset M$ is a neighborhood of $x$. 
It is easy to check that $\stackrel{v}{M}$ is a good topological space containing $M$ as an open subset.

Define $\stackrel{\vee}{p}: \stackrel{\vee}{M} \rightarrow \stackrel{\vee}{\mathrm{S}}$ by $\stackrel{\vee}{p}(j(x))=\stackrel{\circ}{p}(x)$ for $x \in M$, and $\stackrel{\vee}{p}(i(s))=s$ for $s \in$ S. Then, $\stackrel{\vee}{p}$ is proper. It follows that, setting $M_{\infty}:=(M, \stackrel{v}{M})$, the morphism

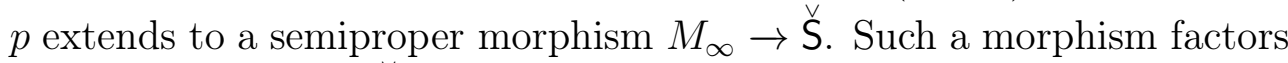
as $M_{\infty} \stackrel{p_{\infty}}{\longrightarrow} \mathrm{S} \stackrel{j_{\mathrm{S}}}{\longrightarrow} \stackrel{\vee}{\mathrm{S}}$, and hence also $p_{\infty}$ is semiproper.

This proves the existence. Uniqueness follows from Lemma 2.1, by considering the commutative diagram

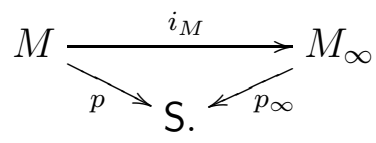

2.3. Blow-ups and normal deformation. Let $M$ be a real analytic manifold and $N \subset M$ a closed submanifold. Denote by $\tau: T_{N} M \rightarrow N$ the normal bundle, and by $\dot{T}_{N} M \subset T_{N} M$ the complement of the zerosection. Recall that the multiplicative groups $\mathbb{R}^{\times}:=\mathbb{R}_{\neq 0}$ and $\mathbb{R}_{>0}^{\times}:=\mathbb{R}_{>0}$ act freely on $\dot{T}_{N} M$. Denote by $S_{N} M:=\dot{T}_{N} M / \mathbb{R}_{>0}^{\times}$the sphere normal bundle, and by $P_{N} M:=\dot{T}_{N} M / \mathbb{R}^{\times}$the projective normal bundle.

Notation 2.4. $\quad$ (i) Denote by $p_{\mathrm{rb}}: M_{N}^{\mathrm{rb}} \rightarrow M$ the real oriented blowup of $M$ with center $N$. Recall that $M_{N}^{\text {rb }}$ is a subanalytic space, that $p_{\mathrm{rb}}$ induces an isomorphism $p_{\mathrm{rb}}^{-1}(M \backslash N) \stackrel{\sim}{\longrightarrow} \backslash \backslash N$, and that $p_{\mathrm{rb}}^{-1}(N)=S_{N} M$. In fact, $M_{N}^{\mathrm{rb}}$ is a real analytic manifold with boundary $S_{N} M$. This is pictured in the commutative diagram

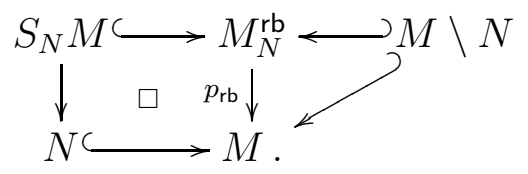

(ii) Denote by $p_{\mathrm{pb}}: M_{N}^{\mathrm{pb}} \rightarrow M$ the real projective blow-up of $M$ with center $N$. Recall that $M_{N}^{\mathrm{pb}}$ is a real analytic manifold, that $p_{\mathrm{pb}}$ induces an isomorphism $p_{\mathrm{pb}}^{-1}(M \backslash N) \stackrel{\sim}{\longrightarrow} M \backslash N$, and that $p_{\mathrm{pb}}^{-1}(N)=P_{N} M$. This is pictured in the commutative diagram

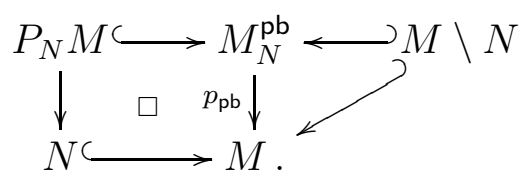

We have a natural commutative diagram

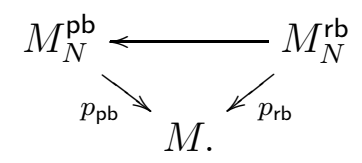


(iii) Denote by $\left(p_{\text {nd }}, s_{\text {nd }}\right): M_{N}^{\text {nd }} \rightarrow M \times \mathbb{R}$ the normal deformation (or deformation to the normal cone) of $M$ along $N$ (see [7, §4.1]). Recall that $M_{N}^{\text {nd }}$ is a real analytic manifold, and that $\left(p_{\text {nd }}, s_{\text {nd }}\right)$ induces isomorphisms $p_{\text {nd }}^{-1}(M \backslash N) \stackrel{\sim}{\longrightarrow}(M \backslash N) \times \mathbb{R}_{\neq 0}$ and $s_{\text {nd }}^{-1}\left(\mathbb{R}_{\neq 0}\right) \stackrel{\sim}{\longrightarrow}$ $M \times \mathbb{R}_{\neq 0}$. One also has $s_{\text {nd }}^{-1}(\{0\})=T_{N} M$. This is pictured in the commutative diagram

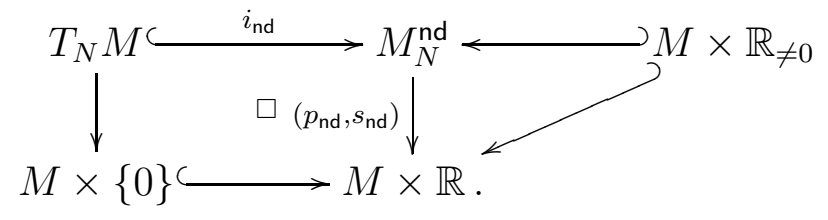

There is a natural action of $\mathbb{R}^{\times}$on $M_{N}^{\text {nd }}$, extending that on $T_{N} M$. The map $s_{\text {nd }}: M_{N}^{\text {nd }} \rightarrow \mathbb{R}$ is smooth and equivariant with respect to the action of $\mathbb{R}^{\times}$on $\mathbb{R}$ given by $c \cdot s=c^{-1} s$. For $\Omega:=s_{\text {nd }}^{-1}\left(\mathbb{R}_{>0}\right) \stackrel{\sim}{\longrightarrow}$ $M \times \mathbb{R}_{>0}$, consider the commutative diagram

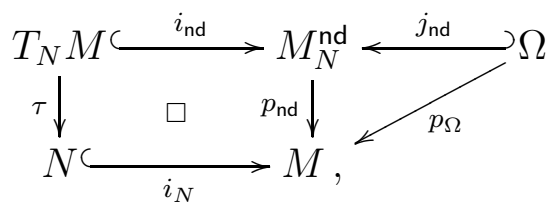

where we set $p_{\Omega}:=\left.p_{\text {nd }}\right|_{\Omega}$. Note that $\bar{\Omega}=s_{\text {nd }}^{-1}\left(\mathbb{R}_{\geqslant 0}\right)=\Omega \sqcup T_{N} M$. For $S \subset M$, the normal cone to $S$ along $N$ is defined by

$$
C_{N}(S):=T_{N} M \cap \overline{p_{\Omega}^{-1}(S)} .
$$

(iv) Denote by $\widetilde{\Omega}$ the complement of $p_{\text {nd }}^{-1}(N) \backslash \dot{T}_{N} M$ in $\bar{\Omega}$, i.e. $\widetilde{\Omega}=$ $\left((M \backslash N) \times \mathbb{R}_{>0}\right) \sqcup \dot{T}_{N} M$. Thus, $\widetilde{\Omega}$ is an open subset of $\bar{\Omega}$ which is invariant by the action of $\mathbb{R}_{>0}^{\times}$, and enters the commutative diagram

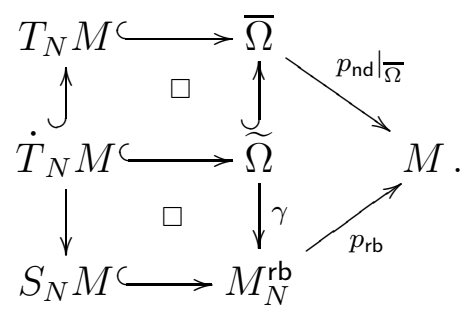

Note that $\gamma: \widetilde{\Omega} \rightarrow M_{N}^{\mathrm{rb}}$ is a principal $\mathbb{R}_{>0}^{\times}$-bundle.

Remark 2.5. Let us illustrate the above constructions in local coordinates. Consider a chart $M \supset U \stackrel{\varphi}{\rightarrow} \mathbb{R}_{x}^{m} \times \mathbb{R}_{y}^{n}$ such that $N \cap U=$ $\varphi^{-1}(\{x=0\})$.

(i) Let $\mathbb{R}_{>0}^{\times}$act on $\mathbb{R}_{v}^{m} \times \mathbb{R}_{y}^{n} \times \mathbb{R}_{s}$ by $c \cdot(v, y, s)=\left(c v, y, c^{-1} s\right)$. Then $p_{\mathrm{rb}}^{-1}(U) \subset M_{N}^{\mathrm{rb}}$ has $\mathbb{R}_{>0}^{\times}$-homogeneous coordinates $[v, y, s]$ with $v \neq 0$, $s \geqslant 0$, and $(s v, y) \in \varphi(U)$. One has $p_{\mathrm{rb}}([v, y, s])=(s v, y)$. 
(ii) Similarly, replacing the action of $\mathbb{R}_{>0}^{\times}$by that of $\mathbb{R}^{\times}$, the open subset $p_{\mathrm{pb}}^{-1}(U) \subset M_{N}^{\mathrm{pb}}$ has $\mathbb{R}^{\times}$-homogeneous coordinates $[v, y, s]$ with $v \neq 0$ and $(s v, y) \in \varphi(U)$. One has $p_{\mathrm{pb}}([v, y, s])=(s v, y)$.

(iii) The open subset $p_{\text {nd }}^{-1}(U) \subset M_{N}^{\text {nd }}$ has coordinates $(v, y, s) \in \mathbb{R}^{m+n+1}$, with $(s v, y) \in \varphi(U)$. One has $p_{\text {nd }}(v, y, s)=(s v, y)$ and $s_{\text {nd }}(v, y, s)=s$. The action of $\mathbb{R}^{\times}$on $M_{N}^{\text {nd }}$ is given by $c \cdot(v, y, s)=\left(c v, y, c^{-1} s\right)$. One has $\Omega \cap p_{\text {nd }}^{-1}(U)=\{s>0\}$ and $\bar{\Omega} \cap p_{\text {nd }}^{-1}(U)=\{s \geqslant 0\}$.

(iv) One has $\widetilde{\Omega} \cap p_{\text {nd }}^{-1}(U)=\{(v, y, s) ; s \geqslant 0, v \neq 0\}$ and $\gamma(v, y, s)=$ $[v, y, s] \in M_{N}^{\mathrm{rb}}$.

2.4. Bordered normal deformation. Let $M$ be a real analytic manifold and $N \subset M$ a closed submanifold. Set $X=M \times \mathrm{P}$ and $Y=$ $N \times\{0\} \subset X$, where $\mathrm{P}:=\mathbb{R} \cup\{\infty\}$ is the real projective line. There is a natural commutative diagram

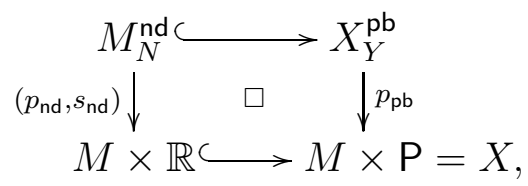

where the bottom arrow is induced by the inclusion of the affine chart $\mathbb{R} \subset$ $\mathrm{P}$, and the top arrow is the embedding described as follows. Recall that $M_{N}^{\text {nd }}=s_{\text {nd }}^{-1}\left(\mathbb{R}_{\neq 0}\right) \sqcup T_{N} M$. The natural identifications $s_{\text {nd }}: s_{\text {nd }}^{-1}\left(\mathbb{R}_{\neq 0}\right) \stackrel{\sim}{\longrightarrow}$ $M \times \mathbb{R}_{\neq 0}$ and $p_{\mathrm{pb}}: p_{\mathrm{pb}}^{-1}(X \backslash Y) \stackrel{\sim}{\longrightarrow}(M \times \mathrm{P}) \backslash(N \times\{0\})$, provide an open embedding $s_{\text {nd }}^{-1}\left(\mathbb{R}_{\neq 0}\right) \subset X_{Y}^{\mathrm{pb}}$. This extends to $M_{N}^{\text {nd }}$ by sending $v \in T_{N} M$ to $[v, 1] \in P_{Y} X=p_{\mathrm{pb}}^{-1}(Y)$. Note that one has

$$
\begin{aligned}
X_{Y}^{\mathrm{pb}} \backslash M_{N}^{\mathrm{nd}} & =p_{\mathrm{pb}}^{-1}(M \times\{\infty\}) \\
=p_{\mathrm{pb}}^{-1}(M \times\{\infty\}) & \sqcup \overline{p_{\mathrm{pb}}^{-1}((M \backslash N) \times\{0\})} \\
& \sqcup p_{\mathrm{pb}}^{-1}((M \backslash N) \times\{0\}) \\
& \sqcup P_{N \times\{0\}}(M \times\{0\}) .
\end{aligned}
$$

Remark 2.6. Let us describe the above constructions in the situation of Remark 2.5. Consider the action of $\mathbb{R}^{\times}$on $\mathbb{R}_{v}^{m} \times \mathbb{R}_{y}^{n} \times \mathbb{R}_{r} \times \mathbb{R}_{s}$ given by $c \cdot(v, y, r, s)=\left(c v, y, c r, c^{-1} s\right)$. Let $\mathbb{R}=\mathrm{P} \backslash\{\infty\}$ be the affine chart. Then $p_{\mathrm{pb}}^{-1}(U \times \mathbb{R}) \subset X_{Y}^{\mathrm{pb}}$ has $\mathbb{R}^{\times}$-homogeneous coordinates $[v, y, r, s]$ with $(v, s) \neq(0,0)$ and $(s v, y) \in \varphi(U)$. One has $p_{\mathrm{pb}}([v, y, r, s])=(s v, y, s r)$. The embedding $M_{N}^{\mathrm{nd}} \hookrightarrow X_{Y}^{\mathrm{pb}}$ is given by $(v, y, s) \mapsto[v, y, 1, s]$.

Recall from $[3, \S 5.4]$ that a real analytic bordered space is a bordered space $M$ such that $\stackrel{v}{M}$ is a real analytic manifold, and $\stackrel{\dot{M}}{\mathrm{C}} \stackrel{\vee}{\mathrm{M}}$ is a subanalytic open subset. A morphism $f: \mathrm{M} \rightarrow \mathrm{N}$ of real analytic bordered spaces is a morphism of bordered spaces such that $f$ is a real analytic map, and $\overline{\Gamma_{f}}$ is a subanalytic subset of $\stackrel{v}{M} \times \stackrel{v}{N}$. 
Lemma-Definition 2.7. The bordered compactification of $M_{N}^{\text {nd }}$ over $M$ has a realization in the category of real analytic bordered spaces by $\left(M_{N}^{\mathrm{nd}}\right)_{\infty}:=\left(M_{N}^{\mathrm{nd}}, X_{Y}^{\mathrm{pb}}\right)$, using the open embedding $(2.7)$. Note that the projection $X_{Y}^{\mathrm{pb}} \rightarrow M$ is proper.

Note that the closure $\overline{T_{N} M}$ of $T_{N} M$ in $P_{Y} X$ is the projective compactification of $T_{N} M$ along the fibers of $\tau: T_{N} M \rightarrow N$. Considering the bordered spaces $\left(T_{N} M\right)_{\infty}:=\left(T_{N} M, \overline{T_{N} M}\right)$ and $\Omega_{\infty}:=\left(\Omega, X_{Y}^{\mathrm{pb}}\right)$, one has the commutative diagram with Cartesian squares of bordered spaces semiproper over $M$

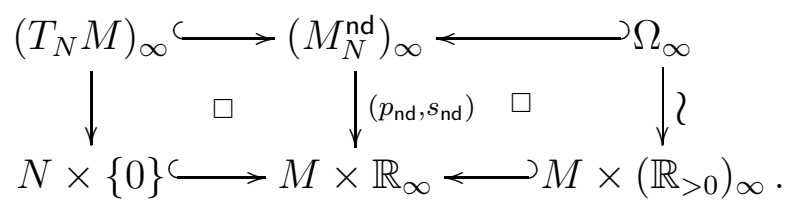

Note that the morphisms in the top row are $\left(\mathbb{R}_{>0}^{\times}\right)_{\infty}$-equivariant. Here, $\left(\mathbb{R}_{>0}^{\times}\right)_{\infty}:=\left(\mathbb{R}_{>0}, \overline{\mathbb{R}}\right)$ is a group object in $b$ - $\mathcal{T}$ op.

\section{REVIEW ON ENHANCED IND-SHEAVES}

We recall here some notions and results, mainly to fix notations, referring to the literature for details. In particular, we refer to [7] for sheaves, to [12] (see also [5, 4]) for enhanced sheaves, to [8] for ind-sheaves, and to [3] (see also [9, 10, 6, 4]) for bordered spaces and enhanced ind-sheaves.

In this paper, $\mathbf{k}$ denotes a base field.

\subsection{Sheaves. Let $M$ be a good space.}

Denote by $\mathrm{D}^{\mathrm{b}}\left(\mathbf{k}_{M}\right)$ the bounded derived category of sheaves of $\mathbf{k}$-vector spaces on $M$, and by $\otimes, f^{-1}, \mathrm{R} f_{\text {! }}$ and $\mathrm{RHom}, \mathrm{R} f_{*}, f^{!}$the six operations. Here $f: M \rightarrow N$ is a morphism of good spaces.

For $S \subset M$ locally closed, we denote by $\mathbf{k}_{S}$ the extension by zero to $M$ of the constant sheaf on $S$ with stalk k.

\subsection{Ind-sheaves. Let $M$ be a bordered space.}

We denote by $\mathrm{D}^{\mathrm{b}}\left(\mathrm{I}_{\mathrm{M}}\right)$ the bounded derived category of ind-sheaves of $\mathrm{k}$-vector spaces on $\mathrm{M}$, and by $\otimes, f^{-1}, \mathrm{R} f_{! !}$and $\mathrm{R} \mathcal{I} h o m, \mathrm{R} f_{*}, f^{!}$the six operations. Here $f: M \rightarrow N$ is a morphism of bordered spaces.

We denote by $\iota_{\mathrm{M}}: \mathrm{D}^{\mathrm{b}}\left(\mathbf{k}_{\mathrm{M}}\right) \rightarrow \mathrm{D}^{\mathrm{b}}\left(\mathrm{I}_{\mathrm{M}}\right)$ the natural embedding, by $\alpha_{\mathrm{M}}$ the left adjoint of $\iota_{\mathrm{M}}$. One sets RHom $:=\alpha_{\mathrm{M}}$ RIhom .

For $F \in \mathrm{D}^{\mathrm{b}}\left(\mathbf{k}_{\mathrm{M}}\right)$, we often write simply $F$ instead of $\iota_{\mathrm{M}} F$ in order to make notations less heavy. 
3.3. Enhanced ind-sheaves. Denote by $t \in \mathbb{R}$ the coordinate on the affine line, consider the two-point compactification $\overline{\mathbb{R}}:=\mathbb{R} \cup\{-\infty,+\infty\}$, and set $\mathbb{R}_{\infty}:=(\mathbb{R}, \overline{\mathbb{R}})$. For $\mathrm{M}$ a bordered space, consider the projection

$$
\pi_{\mathrm{M}}: \mathrm{M} \times \mathbb{R}_{\infty} \rightarrow \mathrm{M}
$$

Denote by $\mathrm{E}^{\mathrm{b}}\left(\mathrm{I} \mathbf{k}_{\mathrm{M}}\right):=\mathrm{D}^{\mathrm{b}}\left(\mathrm{I}_{\mathrm{M} \times \mathbb{R}_{\infty}}\right) / \pi_{\mathrm{M}}^{-1} \mathrm{D}^{\mathrm{b}}\left(\mathrm{I} \mathbf{k}_{\mathrm{M}}\right)$ the bounded derived category of enhanced ind-sheaves of $\mathbf{k}$-vector spaces on $\mathrm{M}$. Denote by

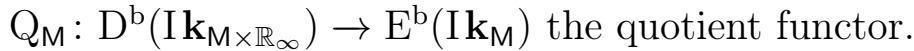

For $f: \mathrm{M} \rightarrow \mathrm{N}$ a morphism of bordered spaces, set

$$
f_{\mathbb{R}}:=f \times \mathrm{id}_{\mathbb{R}_{\infty}}: \mathrm{M} \times \mathbb{R}_{\infty} \rightarrow \mathrm{N} \times \mathbb{R}_{\infty} .
$$

Denote by $\stackrel{+}{\otimes}, \mathrm{E} f^{-1}, \mathrm{E} f_{! !}$and $\mathrm{RI} h o m^{+}, \mathrm{E} f_{*}, \mathrm{E} f^{!}$the six operations for enhanced ind-sheaves. Recall that $\stackrel{+}{\otimes}$ is the additive convolution in the $t$ variable, and that the external operations are induced via $\mathrm{Q}$ by the corresponding operations for ind-sheaves, with respect to the morphism $f_{\mathbb{R}}$. Denote by $\mathrm{D}_{M}^{\mathrm{E}}$ the Verdier dual.

There is a natural decomposition $\mathrm{E}^{\mathrm{b}}\left(\mathrm{I}_{\mathbf{k}_{\mathrm{M}}}\right) \simeq \mathrm{E}_{+}^{\mathrm{b}}\left(\mathrm{I}_{\mathrm{M}}\right) \oplus \mathrm{E}_{-}^{\mathrm{b}}\left(\mathrm{Ik}_{\mathrm{M}}\right)$, there are embeddings

$$
\epsilon_{\mathrm{M}}^{ \pm}: \mathrm{D}^{\mathrm{b}}\left(\mathrm{I}_{\mathrm{M}}\right) \longmapsto \mathrm{E}_{ \pm}^{\mathrm{b}}\left(\mathrm{I}_{\mathrm{M}}\right), \quad F \mapsto \mathrm{Q}_{\mathrm{M}}\left(\mathbf{k}_{\{ \pm t \geqslant 0\}} \otimes \pi_{\mathrm{M}}^{-1} F\right)
$$

and one sets $\epsilon_{\mathrm{M}}(F):=\epsilon_{\mathrm{M}}^{+}(F) \oplus \epsilon_{\mathrm{M}}^{-}(F) \in \mathrm{E}^{\mathrm{b}}\left(\mathrm{I}_{\mathrm{M}}\right)$. Note that $\epsilon_{\mathrm{M}}(F) \simeq$ $\mathrm{Q}_{\mathrm{M}}\left(\mathbf{k}_{\{t=0\}} \otimes \pi_{\mathrm{M}}^{-1} F\right)$.

3.4. Stable objects. Let $M$ be a bordered space. Set

$$
\begin{aligned}
& \mathbf{k}_{\{t \gg 0\}}:=\underset{a \rightarrow+\infty}{\stackrel{\lim }{\longrightarrow}} \mathbf{k}_{\{t \geqslant a\}} \in \mathrm{D}^{\mathrm{b}}\left(\mathrm{I}_{\mathrm{M} \times \mathbb{R}_{\infty}}\right), \\
& \mathbf{k}_{\mathrm{M}}^{\mathrm{E}}:=\mathrm{Q}_{\mathrm{M}} \mathbf{k}_{\{t \gg 0\}} \in \mathrm{E}_{+}^{\mathrm{b}}\left(\mathrm{I}_{\mathrm{M}}\right) \text {. }
\end{aligned}
$$

An object $K \in \mathrm{E}^{\mathrm{b}}\left(\mathrm{I}_{\mathrm{M}}\right)$ is called stable if $K \stackrel{\sim}{\longrightarrow} \mathbf{k}_{\mathrm{M}}^{\mathrm{E}} \stackrel{+}{\otimes} K$.

There is an embedding

$$
e_{\mathrm{M}}: \mathrm{D}^{\mathrm{b}}\left(\mathrm{I}_{\mathrm{M}}\right) \longmapsto \mathrm{E}_{+}^{\mathrm{b}}\left(\mathrm{I}_{\mathrm{M}}\right), \quad F \mapsto \mathbf{k}_{\mathrm{M}}^{\mathrm{E}} \stackrel{+}{\otimes} \epsilon_{\mathrm{M}}(F),
$$

with values in stable objects.

\section{Specialization}

We discuss here the natural enhancement of the notions of conic object and Sato's specialization. For the corresponding classical notions we refer to $[7, \S 3.7]$ and $[7, \S 4.2]$, respectively. We also link the specialization functor with the real oriented blow-up. 
4.1. Conic objects. Recall that the bordered space $\left(\mathbb{R}_{>0}^{\times}\right)_{\infty}:=\left(\mathbb{R}_{>0}, \overline{\mathbb{R}}\right)$ is semiproper and has a structure of bordered group (i.e., is a group object in the category of bordered spaces). Let $M$ be a bordered space endowed with an action of $\left(\mathbb{R}_{>0}^{\times}\right)_{\infty}$, and consider the maps

$$
p, \mu: \mathrm{M} \times\left(\mathbb{R}_{>0}^{\times}\right)_{\infty} \rightarrow \mathrm{M},
$$

where $p$ is the projection and $\mu$ is the action. Similarly to [7], one says that an object $K \in \mathrm{E}^{\mathrm{b}}\left(\mathrm{I}_{\mathrm{M}}\right)$ is $\left(\mathbb{R}_{>0}^{\times}\right)_{\infty}$-conic if there is an isomorphism

$$
\mathrm{E} p^{-1} K \simeq \mathrm{E} \mu^{-1} K \text {. }
$$

(Recall that if $\mathrm{E} p^{-1} K$ and $\mathrm{E} \mu^{-1} K$ are isomorphic, then there exists a unique isomorphism which restricts to the identity on $\mathrm{M} \times\{1\}$.) Denote by $\mathrm{E}_{\left(\mathbb{R}_{>0}^{\times}\right)_{\infty}}^{\mathrm{b}}\left(\mathrm{I} \mathbf{k}_{\mathrm{M}}\right)$ the full triangulated subcategory of conic objects.

We say that a morphism $\gamma: \mathrm{M} \rightarrow S$ is a principal $\left(\mathbb{R}_{>0}^{\times}\right)_{\infty}$-bundle if it is semiproper and if $M$ is endowed with an action of $\left(\mathbb{R}_{>0}^{\times}\right)_{\infty}$ such that the underlying map $\stackrel{\circ}{\gamma}: \stackrel{\circ}{M} \rightarrow S$ is a principal $\mathbb{R}_{>0}^{\times}$-bundle.

Lemma 4.1. Let $\gamma: \mathrm{M} \rightarrow S$ be a principal $\left(\mathbb{R}_{>0}^{\times}\right)_{\infty}$-bundle. Then, for $K \in \mathrm{E}_{\left(\mathbb{R}_{>0}^{\times}\right)_{\infty}}^{\mathrm{b}}\left(\mathrm{I} \mathbf{k}_{\mathrm{M}}\right)$

(i) one has

$$
K \simeq \mathrm{E} \gamma^{-1} \mathrm{E} \gamma_{*} K \simeq \mathrm{E} \gamma^{!} \mathrm{E} \gamma_{! !} K
$$

In particular, $K \simeq \mathrm{E}^{-1} H$ for some $H \in \mathrm{E}^{\mathrm{b}}\left(\mathrm{Ik}_{S}\right)$.

(ii) One has $\mathrm{E} \gamma_{! !} K \simeq \mathrm{E} \gamma_{*} K[-1]$.

Proof. (i) Since the proofs are similar, let us only discuss the first isomorphism. Consider the cartesian diagram

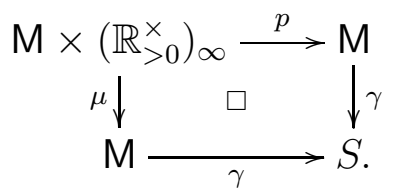

Recalling that $K$ is $\left(\mathbb{R}_{>0}^{\times}\right)_{\infty}$-conic, one has

$$
\mathrm{E} \gamma^{-1} \mathrm{E} \gamma_{*} K \simeq \mathrm{E} \gamma^{!} \mathrm{E} \gamma_{*} K[-1] \simeq \mathrm{E} p_{*} \mathrm{E} \mu^{!} K[-1] \simeq \mathrm{E} p_{*} \mathrm{E} p^{!} K[-1] .
$$

Then, Sublemma 4.2 implies

$$
\begin{aligned}
\mathrm{E}^{-1} \mathrm{E} \gamma_{*} K & \simeq \operatorname{RIhom}^{+}\left(\epsilon_{\mathrm{M}}\left(\mathrm{R} p_{!} \mathbf{k}_{\mathrm{M} \times \mathbb{R}}\right), K\right)[-1] \\
& \simeq \operatorname{RIhom}^{+}\left(\epsilon_{\mathrm{M}}\left(\mathbf{k}_{\mathrm{M}}[-1]\right), K\right)[-1] \simeq K
\end{aligned}
$$

(ii) One has

$$
\mathrm{E} \gamma_{! !} K \simeq \mathrm{E} \gamma_{! !} \mathrm{E} \gamma^{-1} \mathrm{E} \gamma_{*} K \simeq \mathrm{E} \gamma_{*} K \stackrel{+}{\otimes} \epsilon_{S}\left(\mathrm{R} \gamma_{!} \mathbf{k}_{\stackrel{\mathrm{M}}{ }}\right) \simeq \mathrm{E} \gamma_{*} K[-1]
$$

where the first isomorphism follows from (i), and the second isomorphism follows from Sublemma 4.2. 
Sublemma 4.2. Let $f: \mathrm{M} \rightarrow \mathrm{N}$ be a semiproper morphism of bordered spaces. Then, for any $K \in \mathrm{E}^{\mathrm{b}}\left(\mathrm{I}_{\mathrm{M}}\right)$ one has

$$
\begin{aligned}
\mathrm{E} f_{! !} \mathrm{E} f^{-1} K & \simeq K \stackrel{+}{\otimes} \epsilon_{\mathrm{N}}\left(\mathrm{R} f_{!} \mathbf{k}_{\mathrm{M}}\right), \\
\mathrm{E} f_{*} \mathrm{E} f^{!} K & \simeq \mathrm{R} \mathcal{I} h o m^{+}\left(\epsilon_{\mathrm{N}}\left(\mathrm{R} f_{!} \mathbf{k}_{\mathrm{M}}\right), K\right) .
\end{aligned}
$$

Proof. The first isomorphism follows from

$$
\begin{aligned}
\mathrm{E} f_{! !} \mathrm{E} f^{-1} K & \simeq \mathrm{E} f_{! !}\left(\mathrm{E} f^{-1} K \stackrel{+}{\otimes} \epsilon_{\mathrm{M}}\left(\mathbf{k}_{\dot{\mathrm{M}}}\right)\right) \\
& \simeq K \stackrel{+}{\otimes} \mathrm{E} f_{! !}\left(\epsilon_{\mathrm{M}}\left(\mathbf{k}_{\dot{\mathrm{M}}}\right)\right) \\
& \simeq K \stackrel{+}{\otimes} \epsilon_{\mathrm{N}}\left(\mathrm{R} f_{!} \mathbf{k}_{\dot{\mathrm{M}}}\right)
\end{aligned}
$$

where the last isomorphism is due to the fact that $f$ is semiproper.

Similarly, the second isomorphism follows from

$$
\begin{aligned}
\operatorname{E} f_{*} \operatorname{Ef}^{!} K & \simeq \operatorname{E} f_{*} \operatorname{RIhom}^{+}\left(\epsilon_{\mathrm{M}}\left(\mathbf{k}_{\mathrm{M}}\right), \mathrm{E} f^{!} K\right) \\
& \simeq \operatorname{RIhom}^{+}\left(\operatorname{E} f_{! !}\left(\epsilon_{\mathrm{M}}\left(\mathbf{k}_{\dot{M}}\right)\right), K\right) \\
& \simeq \operatorname{RIhom}^{+}\left(\epsilon_{\mathrm{N}}\left(\operatorname{R} f_{!} \mathbf{k}_{\dot{M}}\right), K\right) .
\end{aligned}
$$

4.2. Conic objects on vector bundles. Let $\tau: V \rightarrow N$ be a real vector bundle over a good space $N$, and let $\dot{V}=V \backslash N$ be the complement of the zero-section. Let $S_{N} V \rightarrow N$ be the associated sphere bundle defined by $S_{N} V:=\dot{V} / \mathbb{R}_{>0}^{\times}$. Consider the vector bundle $W:=\mathbb{R} \times V \rightarrow N$, and let $\dot{W}:=W \backslash(\{0\} \times N)$ be the complement of the zero section. The fiberwise sphere compactification $\mathbb{S} V \rightarrow N$ of $V \rightarrow N$ is the quotient $\mathbb{S} V:=\dot{W} / \mathbb{R}_{>0}^{\times}$, where the action is given by $c \cdot(u, x)=(c u, c x)$. The bordered compactification of $V \rightarrow N$ is given by $V_{\infty}=(V, \mathbb{S} V)$. It is endowed with a natural $\left(\mathbb{R}_{>0}^{\times}\right)_{\infty}$-action. Consider the morphisms

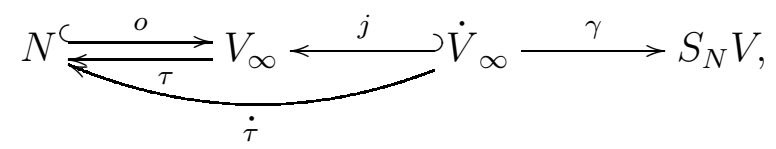

where $o$ is the embedding of the zero section, $j$ is the open embedding, and $\gamma$ the quotient by the action of $\mathbb{R}_{>0}^{\times}$.

Notation 4.3. For $K \in \mathrm{E}_{\left(\mathbb{R}_{>0}^{\times}\right)_{\infty}}^{\mathrm{b}}\left(\mathrm{I}_{V_{\infty}}\right)$, set

$$
K^{\mathrm{sph}}:=\mathrm{E} \gamma_{*} \mathrm{E} j^{-1} K \in \mathrm{E}^{\mathrm{b}}\left(\mathrm{I}_{S_{N} V}\right) .
$$

Lemma 4.4. For $K \in \mathrm{E}_{\left(\mathbb{R}_{>0}^{\times}\right)_{\infty}}^{\mathrm{b}}\left(\mathrm{I}_{V_{\infty}}\right)$, one has the isomorphisms

(i) $\mathrm{E} j^{-1} K \simeq \mathrm{E} \gamma^{-1} K^{\mathrm{sph}}$,

(ii) $\mathrm{E} \tau_{*} K \simeq \mathrm{E}^{-1} K$,

(iii) $\mathrm{E} \tau_{! !} K \simeq \mathrm{E} o^{!} K$ 
and a distinguished triangle

(iv) $\mathrm{E} \dot{\tau}_{! !} \mathrm{E} \gamma^{-1} K^{\mathrm{sph}} \rightarrow \mathrm{E} o^{!} K \rightarrow \mathrm{E}^{-1} K \stackrel{+1}{\longrightarrow}$.

Proof. (i) follows from Lemma 4.1.

(ii) We will adapt some arguments in the proof of [11, Lemma 2.1.12].

One has

$$
\begin{aligned}
& \mathrm{E} \tau_{*} \mathrm{E} o_{*} \mathrm{E} o^{-1} K \simeq \mathrm{E} o^{-1} K, \\
& \mathrm{E} \tau_{*} \mathrm{E} j_{! !} \mathrm{E} j^{-1} K \simeq \mathrm{E} \tau_{*} \mathrm{E} j_{! !} \mathrm{E} \gamma^{-1} \mathrm{E} \gamma_{*} \mathrm{E} j^{-1} K,
\end{aligned}
$$

where the last isomorphism follows from Lemma 4.1. Applying $\mathrm{E} \tau_{*}$ to the distinguished triangle

$$
\mathrm{E} j_{! !} \mathrm{E} j^{-1} K \rightarrow K \rightarrow \mathrm{E} o *_{*} \mathrm{E}^{-1} K \stackrel{+1}{\longrightarrow},
$$

we are thus left to prove

$$
\mathrm{E} \tau_{*} \mathrm{E} j_{! !} \mathrm{E} \gamma^{-1} H \simeq 0
$$

for $H=\mathrm{E} \gamma_{*} \mathrm{E} j^{-1} K$. Let us prove it for an arbitrary $H \in \mathrm{E}^{\mathrm{b}}\left(\mathrm{I}_{S_{N} V}\right)$.

Denoting by $\left(V_{N}^{\mathrm{rb}}\right)_{\infty}:=\left(V_{N}^{\mathrm{rb}}, \mathbb{S} V_{N}^{\mathrm{rb}}\right)$ the bordered compactification of the real oriented blow-up $p_{\mathrm{rb}}: V_{N}^{\mathrm{rb}} \rightarrow V_{\infty}$, consider the commutative diagram

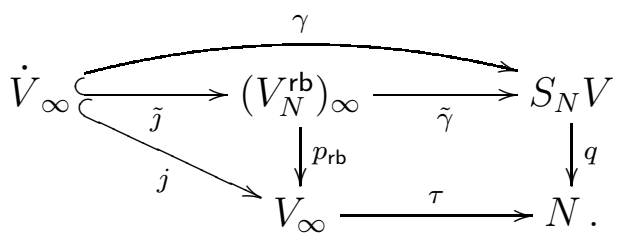

Note that $\tilde{\gamma}: V_{N}^{\mathrm{rb}} \rightarrow S_{N} V,[x, r] \rightarrow[x]$, is an $\mathbb{R}_{\geqslant 0}$-fiber bundle. Hence one has (where we neglect for short the indices on $\epsilon$ and $\iota$ )

$$
\begin{aligned}
\mathrm{E} \tilde{\gamma}^{!} H & \simeq \epsilon\left(\tilde{\gamma}^{!} \mathbf{k}_{S_{N} V}\right) \stackrel{+}{\otimes} \mathrm{E} \tilde{\gamma}^{-1} H \\
& \simeq \epsilon\left(\mathbf{k}_{\tilde{\jmath}(\dot{V})}\right) \stackrel{+}{\otimes} \mathrm{E} \tilde{\gamma}^{-1} H[1], \\
\mathrm{R} \tilde{\gamma}_{!} \mathbf{k}_{V_{N}^{\text {rb }}} & \simeq 0 .
\end{aligned}
$$

Back to (4.1), one has

$$
\begin{aligned}
& \mathrm{E} \tau_{*} \mathrm{E} j_{! !} \mathrm{E} \gamma^{-1} H \simeq \mathrm{E} \tau_{*} \mathrm{E} p_{\mathrm{rb} ! !} \mathrm{E} \tilde{\jmath}_{! !} \mathrm{E} \gamma^{-1} H \\
& \underset{(1)}{\sim} \mathrm{E} \tau_{*} \mathrm{E} p_{\mathrm{rb} *} \mathrm{E} \tilde{\jmath}_{! !} \mathrm{E} \gamma^{-1} H \\
& \simeq \mathrm{E} q_{*} \mathrm{E} \tilde{\gamma}_{*} \mathrm{E} \tilde{\jmath}_{! !} \mathrm{E} \tilde{\jmath}^{-1} \mathrm{E} \tilde{\gamma}^{-1} H \\
& \simeq \mathrm{E} q_{*} \mathrm{E} \tilde{\gamma}_{*}\left(\epsilon\left(\mathbf{k}_{\tilde{\jmath}(\dot{V})}\right) \stackrel{+}{\otimes} \mathrm{E} \tilde{\gamma}^{-1} H\right) \\
& \underset{(2)}{\simeq} \mathrm{E} q_{*} \mathrm{E} \tilde{\gamma}_{*} \mathrm{E} \tilde{\gamma}^{!} H[-1] \\
& \underset{(3)}{\simeq} \mathrm{E}_{*} \mathrm{RI} \operatorname{Ihom}^{+}\left(\epsilon\left(\mathrm{R} \tilde{\gamma}_{!} \mathbf{k}_{V_{N}^{\mathrm{r}}}\right), H\right)[-1] \underset{(4)}{\simeq} 0,
\end{aligned}
$$


where (1) is due to the fact that $p_{\mathrm{rb}}$ is proper, (2) follows from (4.2), (3) follows from Sublemma 4.2 since $\tilde{\gamma}$ is semiproper, and (4) follows from (4.3).

(iii) has a proof similar to (ii).

(iv) Let us show that the distinguished triangle

$$
\mathrm{E} \tau_{! !} \mathrm{E} j_{! !} \mathrm{E} j^{-1} K \rightarrow \mathrm{E} \tau_{! !} K \rightarrow \mathrm{E} \tau_{! !} \mathrm{E} o_{! !} \mathrm{E}^{-1} K \stackrel{+1}{\longrightarrow}
$$

is isomorphic to the distinguished triangle in the statement.

(iv-a) One has $\mathrm{E} \tau_{! !} \mathrm{E} j_{! !} \mathrm{E} j^{-1} K \simeq \mathrm{E} \dot{\tau}_{! !} \mathrm{E} j^{-1} K \simeq \mathrm{E} \dot{\tau}_{! !} \mathrm{E} \gamma^{-1} K^{\text {sph }}$, where the last isomorphism follows from (i).

(iv-b) By (iii), one has $\mathrm{E} \tau ! ! K \simeq \mathrm{E} o ! K$.

(iv-c) One has $\mathrm{E} \tau_{! !} \mathrm{E} o_{! !} \mathrm{E}^{-1} K \simeq \mathrm{E}^{-1} K$, since $\tau \circ o \simeq \mathrm{id}_{N}$.

Lemma 4.5. For $K \in \mathrm{E}_{\left(\mathbb{R}_{>0}^{\times}\right)_{\infty}}^{\mathrm{b}}\left(\mathrm{I}_{V_{\infty}}\right)$, one has

$$
\mathrm{D}^{\mathrm{E}}\left(K^{\mathrm{sph}}\right) \simeq\left(\mathrm{D}^{\mathrm{E}} K\right)^{\mathrm{sph}}[-1]
$$

Proof. One has

$$
\begin{aligned}
\mathrm{D}^{\mathrm{E}}\left(\mathrm{E} \gamma_{*} \mathrm{E} j^{-1} K\right) & \underset{(*)}{\simeq} \mathrm{D}^{\mathrm{E}}\left(\mathrm{E} \gamma_{! !} \mathrm{E} j^{-1} K[1]\right) \\
& \simeq \mathrm{E} \gamma_{*} \mathrm{E} j^{!} \mathrm{D}^{\mathrm{E}}(K)[-1] \\
& \simeq \mathrm{E} \gamma_{*} \mathrm{E} j^{-1} \mathrm{D}^{\mathrm{E}}(K)[-1],
\end{aligned}
$$

where $(*)$ follows from Lemma 4.1 (ii).

4.3. Enhanced specialization. Let $M$ be a real analytic manifold, and $N \subset M$ a closed submanifold. We will introduce here an enhancement of Sato's specialization functor. We refer to [7, Chapter 4] for the classical construction.

Note that the action of $\mathbb{R}_{>0}^{\times}$on (2.3) naturally extends to an action of $\left(\mathbb{R}_{>0}^{\times}\right)_{\infty}$ on its bordered compactification. Consider the morphisms

$$
\left(T_{N} M\right)_{\infty} \stackrel{i_{\text {nd }}}{\longrightarrow}\left(M_{N}^{\text {nd }}\right)_{\infty} \stackrel{j_{\text {nd }}}{\longrightarrow} \Omega_{\infty} \stackrel{p_{\text {nd }} \mid \Omega}{\longrightarrow} M
$$

In the following, when there is no risk of confusion, we will write for short $i=i_{\text {nd }}, j=j_{\text {nd }}$ and $p_{\Omega}=\left.p_{\text {nd }}\right|_{\Omega}$.

Definition 4.6. For $K \in \mathrm{E}^{\mathrm{b}}\left(\mathrm{I}_{\mathbf{k}_{M}}\right)$, we set

$$
\begin{aligned}
\mathrm{E} \nu_{N}(K) & :=\mathrm{E}^{-1} \mathrm{E} j_{*} \mathrm{E}_{p^{-1} K} \in \mathrm{E}_{\left(\mathbb{R}_{>0}^{\times}\right)_{\infty}}^{\mathrm{b}}\left(\mathrm{Ik}_{\left(T_{N} M\right)_{\infty}}\right), \\
\mathrm{E} \nu_{N}^{\mathrm{sph}}(K) & :=\left(\mathrm{E}_{N}(K)\right)^{\mathrm{sph}} \in \mathrm{E}^{\mathrm{b}}\left(\mathrm{I}_{S_{N} M}\right) .
\end{aligned}
$$

The functor $\mathrm{E} \nu_{N}$ is called enhanced specialization along $N$.

With a proof similar to that of Lemma 4.12 (or of [7, Lemma 4.2.1]), one has 
Lemma 4.7. For $K \in \mathrm{E}^{\mathrm{b}}\left(\mathrm{Ik}_{M}\right)$, one has

$$
\mathrm{E} \nu_{N}(K) \simeq \mathrm{E} i^{!} \mathrm{E} j_{! !} \mathrm{E} p_{\Omega}^{!} K
$$

Note that there is an isomorphism

$$
e \circ \nu_{N} \simeq \mathrm{E} \nu_{N} \circ e
$$

and similarly for $e$ replaced by $\epsilon, \epsilon^{+}$or $\epsilon^{-}$.

Consider the morphisms

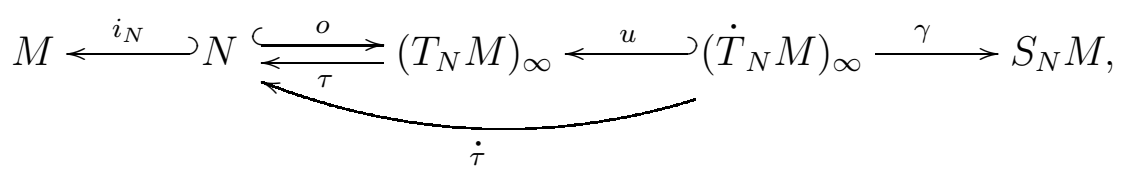

where $o$ is the zero-section.

Lemma 4.8. For $K \in \mathrm{E}^{\mathrm{b}}\left(\mathrm{I}_{M}\right)$, one has the isomorphisms

(i) $\mathrm{E} \tau_{*} \mathrm{E} \nu_{N}(K) \simeq \mathrm{E} o^{-1} \mathrm{E} \nu_{N}(K) \simeq \mathrm{E} i_{N}^{-1} K$,

(ii) $\mathrm{E} \tau_{!} \mathrm{E} \nu_{N}(K) \simeq \mathrm{E} o ! \mathrm{E} \nu_{N}(K) \simeq \mathrm{E} i !$

and distinguished triangles

(iii) $\mathrm{E} \dot{\tau}_{! !} \mathrm{E} u^{-1} \mathrm{E} \nu_{N} K \rightarrow \mathrm{E} i_{N}^{!} K \rightarrow \mathrm{E}_{N}^{-1} K \stackrel{+1}{\rightarrow}$,

(iv) $\mathrm{E} u_{! !} \mathrm{E} \gamma^{-1} \mathrm{E} \nu_{N}^{\text {sph }} K \rightarrow \mathrm{E} \nu_{N} K \rightarrow \mathrm{E} o_{*} \mathrm{E} i_{N}^{-1} K \stackrel{+1}{\longrightarrow}$,

(v) $\mathrm{E} o_{*} \mathrm{E} i_{N}^{!} K \rightarrow \mathrm{E} \nu_{N} K \rightarrow \mathrm{E} u_{*} \mathrm{E} \gamma^{!} \mathrm{E} \nu_{N}^{\text {sph }} K \stackrel{+1}{\longrightarrow}$.

Proof. (i-a) The isomorphism $\mathrm{E} \tau_{*} \mathrm{E} \nu_{N}(K) \simeq \mathrm{E} o^{-1} \mathrm{E} \nu_{N}(K)$ follows from Lemma 4.4 (ii).

(i-b) Let us show that the composition

$$
\mathrm{E} \tau_{*} \mathrm{E} \nu_{N}(K) \rightarrow \mathrm{E} \tau_{*} \mathrm{E} \nu_{N}\left(\mathrm{E} i_{N *} \mathrm{E} i_{N}^{-1} K\right) \stackrel{\mathrm{E} i_{N}^{-1} K}{ }
$$

is an isomorphism. Since the problem is local on $N$, we may work in coordinates as in Remark 2.5 (iii).

Recall that $\bar{\Omega}=s_{\text {nd }}^{-1}\left(\mathbb{R}_{\geqslant 0}\right)=\Omega \sqcup T_{N} M$ is the closure of $\Omega$ in $M_{N}^{\text {nd }}$, and consider the map $r: \bar{\Omega} \rightarrow M \times \mathbb{R}$ given by $r(v, y, s)=(s v, y, s-|v|)$. Then $r$ is a proper map since, in the commutative diagram

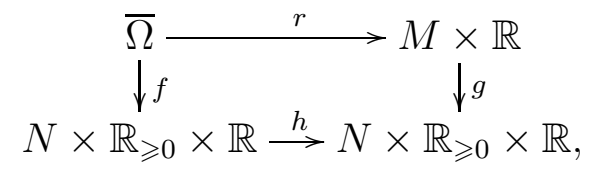

$f$ and $h$ are proper. Here, $f(v, y, s)=(y,|v|, s), g(x, y, s)=(y,|x|, s)$, and $h(y, u, s)=(y, s u, s-u)$.

Setting $Z=N \times \mathbb{R}_{\leqslant 0} \subset M \times \mathbb{R}$ and $U=(M \times \mathbb{R}) \backslash Z$, the continuous map $r$ induces a homeomorphism $\Omega \sim U$. Consider the commutative 
diagram of bordered spaces semiproper over $M$, whose two top squares are cartesian,

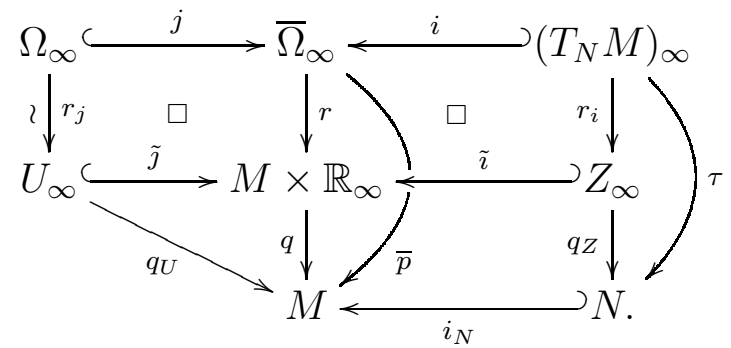

Here, $q, q_{U}$ and $q_{Z}$ denote the first projections, and $\bar{p}=p_{\text {nd }} \mid \bar{\Omega}$. One has

$$
\begin{aligned}
\mathrm{E} \tau_{*} \mathrm{E} \nu_{N}(K) & \simeq \mathrm{E} \tau_{*} \mathrm{E} i^{-1} \mathrm{E} j_{*} \mathrm{E} j^{-1} \mathrm{E} \bar{p}^{-1} K \\
& \simeq \mathrm{E} q_{Z_{*}} \mathrm{E} r_{i *} \mathrm{E} i^{-1} \mathrm{E} j_{*} \mathrm{E} j^{-1} \mathrm{E} r^{-1} \mathrm{E} q^{-1} K \\
& \simeq \mathrm{E} q_{Z_{*}} \mathrm{E} r_{i *} \mathrm{E} i^{-1} \mathrm{E} j_{*} \mathrm{E} r_{j}^{-1} \mathrm{E} \tilde{\jmath}^{-1} \mathrm{E} q^{-1} K \\
& \simeq \mathrm{E} q_{Z_{*}} \mathrm{E} \tilde{\imath}^{-1} \mathrm{E} r_{*} \mathrm{E} j_{*} \mathrm{E} r_{j}^{-1} \mathrm{E} \tilde{\jmath}^{-1} \mathrm{E} q^{-1} K \\
& \simeq \mathrm{E} q_{Z_{*}} \mathrm{E} \tilde{\imath}^{-1} \mathrm{E} \tilde{\jmath}_{*} \mathrm{E} r_{j_{*}} \mathrm{E} r_{j}^{-1} \mathrm{E} \tilde{\jmath}^{-1} \mathrm{E} q^{-1} K \\
& \simeq \mathrm{E} q_{Z_{*}} \mathrm{E} \tilde{\imath}^{-1} \mathrm{E} \tilde{\jmath}_{*} \mathrm{E} \tilde{\jmath}^{-1} \mathrm{E} q^{-1} K,
\end{aligned}
$$

where $(*)$ follows from the properness of $r$. Consider the commutative diagram

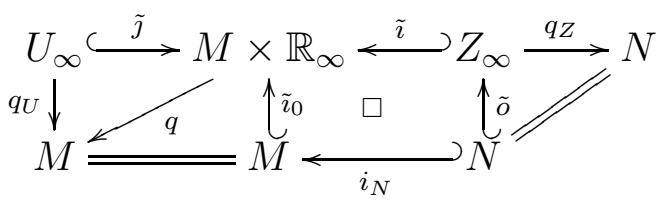

where $\tilde{o}(y)=(0, y, 0)$ and $\tilde{i}_{0}(x, y)=(x, y, 0)$. Note that $\left(\mathbb{R}_{>0}^{\times}\right)_{\infty}$ acts on the second component of $M \times \mathbb{R}_{\infty}$, and hence also on $Z_{\infty}$. Then

$$
\begin{aligned}
\mathrm{E} \tau_{*} \mathrm{E} \nu_{N}(K) \underset{(*)}{\simeq} \mathrm{E} \tilde{o}^{-1} \mathrm{E} \tilde{\imath}^{-1} \mathrm{E} \tilde{\jmath}_{*} \mathrm{E} \tilde{\jmath}^{-1} \mathrm{E} q^{-1} K \\
\simeq \mathrm{E} i_{N}^{-1} \mathrm{E} \tilde{\imath}_{0}^{-1} \mathrm{E} \tilde{\jmath}_{*} \mathrm{E} \tilde{\jmath}^{-1} \mathrm{E} q^{-1} K \\
\underset{(* *)}{\simeq} \mathrm{E} i_{N}^{-1} \mathrm{E} q_{*} \mathrm{E} \tilde{\jmath}_{*} \mathrm{E} \tilde{\jmath}^{-1} \mathrm{E} q^{-1} K \\
\simeq \mathrm{E} i_{N}^{-1} \mathrm{E} q_{U} \mathrm{E} q_{U}^{-1} K,
\end{aligned}
$$


where $(*)$ holds since $\mathrm{E} \tilde{\imath}^{-1} \mathrm{E} \tilde{\jmath}_{*} \mathrm{E} \tilde{\jmath}^{-1} \mathrm{E} q^{-1} K$ is $\left(\mathbb{R}_{>0}^{\times}\right)_{\infty}$-conic, and $(* *)$ holds since $\mathrm{E} \tilde{\jmath}_{*} \mathrm{E} \tilde{\jmath}^{-1} \mathrm{E} q^{-1} K$ is $\left(\mathbb{R}_{>0}^{\times}\right)_{\infty}$-conic. Then, one has

$$
\begin{aligned}
\mathrm{E} \tau_{*} \mathrm{E}_{N}(K) & \simeq \mathrm{E}_{N}^{-1} \mathrm{E} q_{U *} \operatorname{RIhom}^{+}\left(\epsilon\left(\mathbf{k}_{U}\right), \mathrm{E}\left(q_{U}\right) ! K[-1]\right) \\
& \simeq \mathrm{E} i_{N}^{-1} \operatorname{RIhom}^{+}\left(\mathrm{E}_{U ! !} \epsilon\left(\mathbf{k}_{U}\right), K[-1]\right) \\
& \underset{(*)}{ } \mathrm{E}_{N}^{-1} \mathrm{RIhom}^{+}\left(\epsilon\left(\mathrm{R} q_{U !} \mathbf{k}_{U}\right), K[-1]\right) \\
& \underset{(* *)}{\simeq} \mathrm{E}_{N}^{-1} \operatorname{RIhom}^{+}\left(\epsilon\left(\mathbf{k}_{M}[-1]\right), K[-1]\right) \\
& \simeq \mathrm{E} i_{N}^{-1} K,
\end{aligned}
$$

where $(*)$ holds since $q_{U}$ is semiproper, and $(* *)$ is due to the fact that the fibers of $q_{U}$ are homeomorphic to $\mathbb{R}$. This gives (4.5).

(ii) has a similar proof to (i).

(iii) follows from (i) and (ii), using Lemma 4.4 (iv).

(iv) Consider the distinguished triangle

$$
\mathrm{E} u_{! !} \mathrm{E}^{-1} \mathrm{E} \nu_{N}(K) \rightarrow \mathrm{E} \nu_{N}(K) \rightarrow \mathrm{E} o ! ! \mathrm{E}^{-1} \mathrm{E} \nu_{N}(K) \stackrel{+1}{\longrightarrow} .
$$

Then the statement follows from (i) and Lemma 4.4 (i).

(v) has a proof similar to that of (iv), using the distinguished triangle

$$
\mathrm{E} o_{*} \mathrm{E} o ! \mathrm{E} \nu_{N}(K) \rightarrow \mathrm{E} \nu_{N}(K) \rightarrow \mathrm{E} u_{*} \mathrm{E} u ! \mathrm{E} \nu_{N}(K) \stackrel{+1}{\rightarrow} .
$$

Recall the definition of the normal cone in (2.5). Here is an analogue of [7, Exercise IV.2]

Lemma 4.9. Let $S \subset M$ be a closed subset. Then $\mathrm{E} \nu_{N}$ induces a functor $\mathrm{E} \nu_{N \mid M \backslash S}$ (see (4.6) below) entering the quasi-commutative diagram

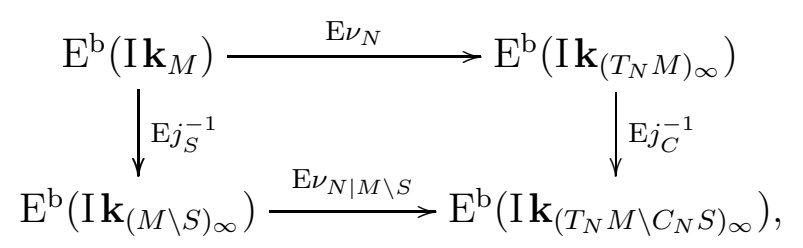

where $j_{S}:(M \backslash S)_{\infty} \rightarrow M$ and $j_{C}:\left(T_{N} M \backslash C_{N} S\right)_{\infty} \rightarrow\left(T_{N} M\right)_{\infty}$ are the open embeddings.

Proof. Consider the commutative diagram with cartesian squares

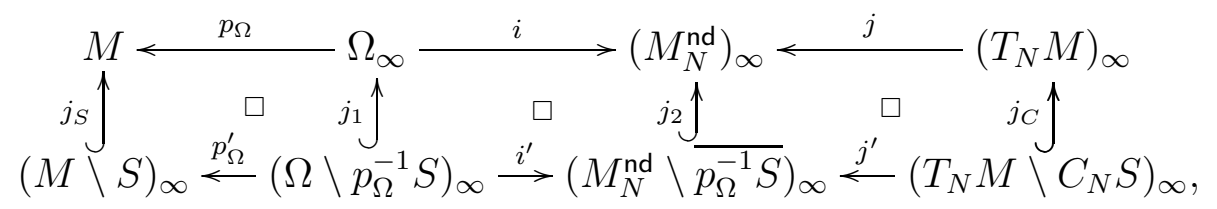


where all the vertical arrows are open embeddings. Then

$$
\begin{aligned}
\mathrm{E} j_{C}^{-1} \mathrm{E} \nu_{N}(K) & =\mathrm{E} j_{C}^{-1} \mathrm{E} j_{*} \mathrm{E} i_{*} \mathrm{E} p_{\Omega}^{-1} K \\
& \simeq \mathrm{E} j_{*}^{\prime} \mathrm{E} i_{*}^{\prime} \mathrm{E} p_{\Omega}^{\prime-1} \mathrm{E} j_{S}^{-1} K .
\end{aligned}
$$

For $K^{\prime} \in \mathrm{E}^{\mathrm{b}}\left(\mathrm{I}_{(M \backslash S)_{\infty}}\right)$, set

$$
\mathrm{E} \nu_{N \mid M \backslash S}\left(K^{\prime}\right):=\mathrm{E} j_{*}^{\prime} \mathrm{E} i_{*}^{\prime} \mathrm{E}_{\Omega}^{\prime-1} K^{\prime} .
$$

Then the statement is clear.

Here is an analogue of [7, Exercise IV.5]

Lemma 4.10. Let $\tau: V \rightarrow N$ be a vector bundle, and denote by $o: N \rightarrow$ $V$ the embedding of the zero-section. For $K \in \mathrm{E}_{\left(\mathbb{R}_{>0}^{\times}\right)_{\infty}}^{\mathrm{b}}\left(\mathrm{I}_{V_{\infty}}\right)$, one has

$$
\mathrm{E} \nu_{N}(K) \simeq K
$$

where we use the identifications $N \simeq o(N) \subset V$ and $T_{N} V \simeq V$.

Proof. One has $\left(V_{N}^{\text {nd }}\right)_{\infty} \simeq V \times \mathbb{R}_{\infty}$, with $s_{\text {nd }}(x, s)=s$ and $p_{\text {nd }}(x, s)=s x$. Hence $\Omega_{\infty} \simeq V \times\left(\mathbb{R}_{>0}^{\times}\right)_{\infty}$, and $p_{\Omega}=\mu$, where $\mu$ is the $\left(\mathbb{R}_{>0}^{\times}\right)_{\infty}$-action. Then,

$$
\mathrm{E} p_{\Omega}^{-1} K=\mathrm{E} \mu^{-1} K \simeq K \stackrel{+}{\otimes} e_{\mathbb{R}_{\infty}}\left(\mathbf{k}_{\mathbb{R}_{>0}}\right),
$$

where the last isomorphism is due to the fact that $K$ is $\left(\mathbb{R}_{>0}^{\times}\right)_{\infty}$-conic. Recalling Definition 4.6, the statement easily follows.

Let $f: M_{1} \rightarrow M_{2}$ be a morphism of real analytic manifolds, let $N_{i} \subset M_{i}$ $(i=1,2)$ be closed submanifolds, and assume $f\left(N_{1}\right) \subset N_{2}$. Consider the associated morphism, given by the composition

$$
T_{N_{1}} f:\left(T_{N_{1}} M_{1}\right)_{\infty} \stackrel{f^{\prime}}{\rightarrow} N_{1} \times_{N_{2}}\left(T_{N_{2}} M_{2}\right)_{\infty} \rightarrow\left(T_{N_{2}} M_{2}\right)_{\infty} .
$$

The enhanced specialization functor satisfies the analogous functorial properties as those in Propositions 4.2.4, 4.2.5 and 4.2.6 of [7]. The proofs in loc. cit. immediately extend to the enhanced framework.

For example, if $f$ and $\left.f\right|_{N_{1}}: N_{1} \rightarrow N_{2}$ are smooth, one has

$$
\mathrm{E}\left(T_{N_{1}} f\right)^{-1} \circ \mathrm{E} \nu_{N_{2}} \simeq \mathrm{E} \nu_{N_{1}} \circ \mathrm{E} f^{-1} .
$$

4.4. Blow-up transform. Let $M$ be a real analytic manifold, and $N \subset$ $M$ a closed submanifold. With notations as in (2.1), consider the real oriented blowup $M_{N}^{\mathrm{rb}}$ and the commutative diagram of bordered spaces

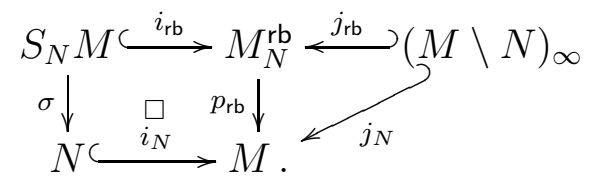

Note that $(M \backslash N)_{\infty} \simeq\left(M_{N}^{\mathrm{rb}} \backslash S_{N} M\right)_{\infty}$. In the following, when there is no risk of confusion, we will write for short $i=i_{\mathrm{rb}}, j=j_{\mathrm{rb}}$ and $p=p_{\mathrm{rb}}$. 
Definition 4.11. For $K \in \mathrm{E}^{\mathrm{b}}\left(\mathrm{I}_{M}\right)$, consider the object

$$
\mathrm{E} \nu_{N}^{\mathrm{rb}}(K):=\mathrm{E} i^{-1} \mathrm{E} j_{*} \mathrm{E} j_{N}^{-1} K \quad \in \mathrm{E}^{\mathrm{b}}\left(\mathrm{Ik}_{S_{N} M}\right) .
$$

We denote by

$$
\nu_{N}^{\mathrm{rb}}: \mathrm{D}^{\mathrm{b}}\left(\mathbf{k}_{M}\right) \rightarrow \mathrm{D}^{\mathrm{b}}\left(\mathbf{k}_{S_{N} M}\right)
$$

the analogous functor for sheaves.

Note that, by definition, $\mathrm{E} \nu_{N}^{\mathrm{rb}}$ factors through a functor, that we denote by the same name,

$$
\mathrm{E} \nu_{N}^{\mathrm{rb}}: \mathrm{E}^{\mathrm{b}}\left(\mathrm{I}_{(M \backslash N)_{\infty}}\right) \rightarrow \mathrm{E}^{\mathrm{b}}\left(\mathrm{I}_{S_{N} M}\right) .
$$

Note also that one has

$$
e \circ \nu_{N}^{\mathrm{rb}} \simeq \mathrm{E} \nu_{N}^{\mathrm{rb}} \circ e,
$$

and similarly for $e$ replaced by $e \circ \iota, \epsilon, \epsilon^{+}$or $\epsilon^{-}$.

Lemma 4.12. For $K \in \mathrm{E}^{\mathrm{b}}\left(\mathrm{I}_{M}\right)$, one has

$$
\mathrm{E} \nu_{N}^{\mathrm{rb}}(K) \simeq \mathrm{E} i ! \mathrm{E} j_{! !} \mathrm{E} j_{N}^{!} K[1] .
$$

Proof. For $L \in \mathrm{E}^{\mathrm{b}}\left(\mathrm{I}_{M_{N}^{\mathrm{rb}}}\right)$, there is a distinguished triangle

$$
\mathrm{E} i_{*} \mathrm{E} i ! L \rightarrow L \rightarrow \mathrm{E} j_{*} \mathrm{E} j ! L \stackrel{+1}{\longrightarrow} .
$$

When $L=\mathrm{E} j_{!} \mathrm{E} j_{N}^{-1} K$, the above distinguished triangle reads

$$
\mathrm{E} i_{*} \mathrm{E} i ! \mathrm{E} j_{! !} \mathrm{E} j_{N}^{!} K \rightarrow \mathrm{E} j_{! !} \mathrm{E} j_{N}^{-1} K \rightarrow \mathrm{E} j_{*} \mathrm{E} j_{N}^{-1} K \stackrel{+1}{\rightarrow} .
$$

The statement follows by applying $\mathrm{E} i^{-1}$, and noticing that $\mathrm{E} i^{-1} \mathrm{E} j_{! !} \simeq$ 0 .

Lemma 4.13. For $K \in \mathrm{E}^{\mathrm{b}}\left(\mathrm{I}_{M}\right)$, one has

$$
\mathrm{E} \nu_{N}^{\mathrm{sph}}(K) \simeq \mathrm{E} \nu_{N}^{\mathrm{rb}}(K)
$$

Proof. Considering the morphisms

$$
\left(T_{N} M\right)_{\infty} \stackrel{u}{\longleftrightarrow}\left(\dot{T}_{N} M\right)_{\infty} \stackrel{\gamma}{\longrightarrow} S_{N} M,
$$

it is equivalent to prove

$$
\mathrm{E} u^{-1} \mathrm{E} \nu_{N}(K) \simeq \mathrm{E} \gamma^{-1} \mathrm{E} \nu_{N}^{\mathrm{rb}}(K) .
$$

Consider the commutative diagram, extending (2.6), whose squares are Cartesian with smooth vertical arrows

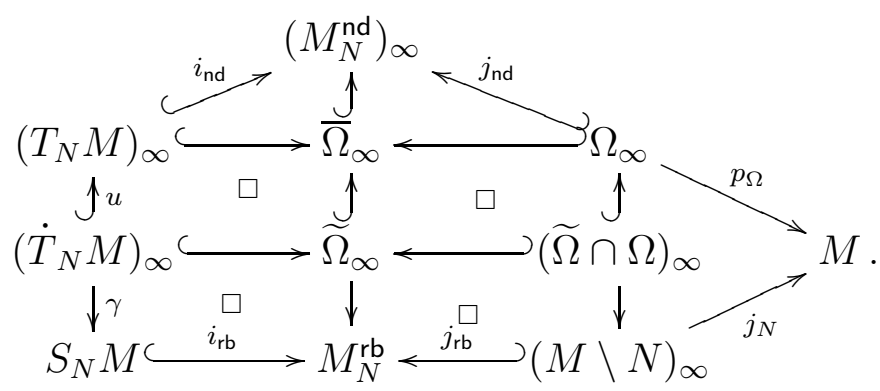


We have to prove

$$
\mathrm{E} u^{-1} \mathrm{E} i_{\text {nd }}^{-1} \mathrm{E} j_{\text {nd } *} \mathrm{E} p_{\Omega}^{-1} K \simeq \mathrm{E} \gamma^{-1} \mathrm{E} i_{\mathrm{rb}}^{-1} \mathrm{E} j_{\mathrm{rb} *} \mathrm{E} j_{N}^{-1} K .
$$

This is obtained by chasing the above diagram.

\section{Fourier-Sato transform AND microlocalization}

We recall here the natural enhancement of the Fourier-Sato transform from $[12, \S 3]$ (see also [1] and [9]), referring to [7, §3.7] for the classical case. We then define the natural enhancement of Sato's microlocalization, for which we refer to $[7, \S 4.3]$.

5.1. Kernels. Let $X$ and $Y$ be bordered spaces. A kernel from $X$ to $Y$ is a triple $(p, q, C)$, where $p$ and $q$ are morphisms of bordered spaces

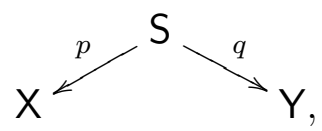

and $C \in \mathrm{E}^{\mathrm{b}}\left(\mathrm{Ik}_{\mathrm{S}}\right)$. To such a kernel one associates the functors

$$
\Phi_{(p, q, C)}, \Psi_{(p, q, C)}: \mathrm{E}^{\mathrm{b}}\left(\mathrm{I}_{\mathbf{X}}\right) \rightarrow \mathrm{E}^{\mathrm{b}}\left(\mathrm{I}_{\mathbf{Y}}\right),
$$

defined by

$$
\begin{aligned}
& \Phi_{(p, q, C)}(K):=\mathrm{E} q_{! !}\left(C \stackrel{+}{\otimes} \mathrm{E}^{-1} K\right), \\
& \Psi_{(p, q, C)}(K):=\mathrm{E}_{*} \operatorname{RIhom}^{+}\left(C, \mathrm{E}^{\prime}{ }^{!} K\right) .
\end{aligned}
$$

Given a commutative diagram

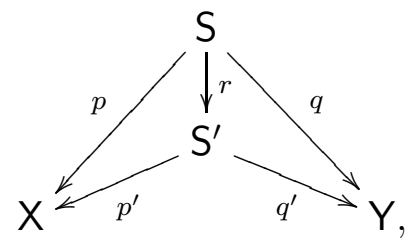

one has

$$
\left.\Phi_{(p, q, C)} \simeq \Phi_{\left(p^{\prime}, q^{\prime}, \mathrm{R} r ! ! C\right)}, \quad \Psi_{(p, q, C)} \simeq \Psi_{\left(p^{\prime}, q^{\prime}, \mathrm{R} r ! !\right.} C\right) .
$$

If there is no fear of confusion, we will write for short

$$
C=(p, q, C), \quad C^{\text {prod }}=\left(q_{1}, q_{2}, \mathrm{R}(p, q) ! C\right), \quad C^{r}=(q, p, C),
$$

where $q_{1}$ and $q_{2}$ are the projections from $\mathrm{X} \times \mathrm{Y}$ to $\mathrm{X}$ and $\mathrm{Y}$, respectively, and $(p, q): \mathbf{S} \rightarrow \mathbf{X} \times \mathbf{Y}$ is the morphism induced by $p$ and $q$. Then, (5.1) implies

$$
\Phi_{C} \simeq \Phi_{C \text { prod }}, \quad \Psi_{C} \simeq \Psi_{C \text { prod }},
$$

and the kernel $C^{r}$ from $\mathrm{Y}$ to $\mathrm{X}$ gives functors

$$
\Phi_{C^{r}}, \Psi_{C^{r}}: \mathrm{E}^{\mathrm{b}}\left(\mathrm{I}_{\mathbf{Y}}\right) \rightarrow \mathrm{E}^{\mathrm{b}}\left(\mathrm{I}_{\mathbf{X}}\right) .
$$


Note that $\Phi_{C}$ is left adjoint to $\Psi_{C^{r}}$ (and $\Psi_{C}$ is right adjoint to $\Phi_{C^{r}}$ ). Note also that for $K \in \mathrm{E}^{\mathrm{b}}\left(\mathrm{I}_{\mathrm{X}}\right)$ one has

$$
\mathrm{D}_{\mathrm{Y}}^{\mathrm{E}} \Phi_{C}(K) \simeq \Psi_{C}\left(\mathrm{D}_{\mathrm{X}}^{\mathrm{E}} K\right)
$$

Note that, if $C \in \mathrm{E}_{ \pm}^{\mathrm{b}}\left(\mathrm{I}_{\mathrm{S}}\right)$, then $\Phi_{C}$ and $\Psi_{C}$ take value in $\mathrm{E}_{ \pm}^{\mathrm{b}}\left(\mathrm{I}_{\mathrm{Y}}\right)$. In this case, we set

$$
\Phi_{C}^{ \pm}:=\left.\Phi_{C}\right|_{\mathrm{E}_{ \pm}^{\mathrm{b}}\left(\mathrm{I} \mathbf{k}_{\mathrm{x}}\right)}, \quad \Psi_{C}^{ \pm}:=\left.\Psi_{C}\right|_{\mathrm{E}_{ \pm}^{\mathrm{b}}\left(\mathrm{I} \mathbf{k}_{\mathrm{x}}\right)},
$$

so that we have functors

$$
\Phi_{C}^{ \pm}, \Psi_{C}^{ \pm}: \mathrm{E}_{ \pm}^{\mathrm{b}}\left(\mathrm{Ik}_{\mathrm{Y}}\right) \rightarrow \mathrm{E}_{ \pm}^{\mathrm{b}}\left(\mathrm{Ik}_{\mathrm{X}}\right) .
$$

For $* \in\{\varnothing,+,-\}$, consider the kernel $\mathbf{1}_{\mathbf{X}}^{*}:=\left(q_{1}, q_{2}, \epsilon^{*}\left(\mathbf{k}_{\Delta_{X}}\right)\right)$, where $X=\stackrel{\circ}{\mathrm{X}}$ and $\Delta_{X} \subset X \times X$ is the diagonal. Note that one has $\mathbf{1}_{\mathrm{X}}^{*} \simeq$ $\left(\operatorname{id}_{\mathbf{X}}, \operatorname{id}_{\mathbf{X}}, \epsilon^{*}\left(\mathbf{k}_{X}\right)\right)^{\text {prod }}$, so that in particular

$$
\Psi_{1_{\mathrm{x}}^{*}}^{*} \simeq \mathrm{id}_{\mathrm{E}_{*}^{\mathrm{b}}\left(\mathrm{Ik}_{\mathrm{x}}\right)} \simeq \Phi_{\mathbf{1}_{\mathrm{X}}^{*}}^{*} .
$$

Given another bordered space $\mathrm{Z}$, and a kernel $D=(\mathrm{T} \stackrel{r}{\rightarrow} \mathrm{Y}, \mathrm{T} \stackrel{s}{\rightarrow} \mathrm{Z}, D)$ from $Y$ to $Z$, consider the diagram with cartesian square

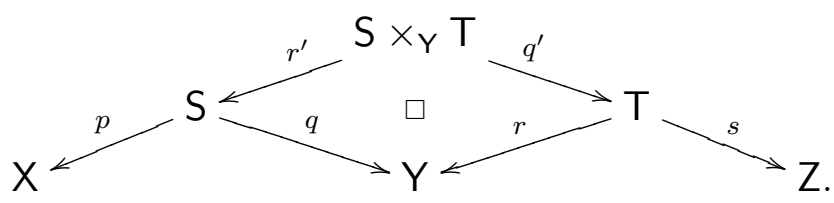

Setting

$$
C \stackrel{+}{\circ} D:=\mathrm{E}^{\prime-1} C \stackrel{+}{\otimes} \mathrm{E}^{\prime-1} D \in \mathrm{E}^{\mathrm{b}}\left(\mathrm{Ik}_{\mathrm{S}_{\times \mathrm{Y}} \mathrm{T}}\right),
$$

one gets a kernel $C \stackrel{+}{\circ} D=\left(p \circ r^{\prime}, s \circ q^{\prime}, C \stackrel{+}{\circ} D\right)$ from $\mathrm{X}$ to $\mathrm{Z}$ such that

$$
\Phi_{D} \circ \Phi_{C} \simeq \Phi_{C \circ D}^{+}, \quad \Psi_{D} \circ \Psi_{C} \simeq \Psi_{C_{\circ D}^{+}} .
$$

Let $* \in\{\varnothing,+,-\}, C \in \mathrm{E}_{*}^{\mathrm{b}}\left(\mathrm{I}_{\mathrm{S}}\right)$ and $D \in \mathrm{E}_{*}^{\mathrm{b}}\left(\mathrm{I}_{\mathrm{T}}\right)$. Assume that $\mathrm{Z}=\mathrm{X}$ and that

$$
(C \stackrel{+}{\circ} D)^{\mathrm{prod}} \simeq \mathbf{1}_{\mathrm{X}}^{*}, \quad\left(D^{+} C\right)^{\mathrm{prod}} \simeq \mathbf{1}_{\mathrm{Y}}^{*} .
$$

Then, the functors $\Phi_{C}^{*}$ and $\Phi_{D}^{*}$ (resp. $\Psi_{C}^{*}$ and $\Psi_{D}^{*}$ ) are equivalences of categories quasi-inverse to each other. Moreover, by uniqueness of the adjoint, one has

$$
\Phi_{C}^{*} \simeq \Psi_{D^{r}}^{*}, \quad \Psi_{C}^{*} \simeq \Phi_{D^{r}}^{*} .
$$

Lemma 5.1. Consider a commutative diagram of bordered spaces with cartesian squares

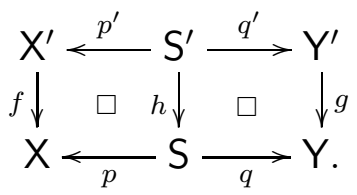


Let $C \in \mathrm{E}^{\mathrm{b}}\left(\mathrm{I}_{\mathrm{s}}\right)$, and set

$$
C^{\prime}:=\mathrm{E}^{-1} C \in \mathrm{E}^{\mathrm{b}}\left(\mathrm{I}_{\mathbf{S}^{\prime}}\right) .
$$

Consider $C=(p, q, C)$ and $C^{\prime}=\left(p^{\prime}, q^{\prime}, C^{\prime}\right)$ as kernels. Then, one has

$$
\begin{aligned}
\Phi_{C} \circ \mathrm{E} f_{! !} & \simeq \mathrm{E} g_{! !} \circ \Phi_{C^{\prime}}, & \Psi_{C} \circ \mathrm{E} f_{*} & \simeq \mathrm{E} g_{*} \circ \Psi_{C^{\prime}}, \\
\Phi_{C^{\prime}} \circ \mathrm{E} f^{-1} & \simeq \mathrm{E} g^{-1} \circ \Phi_{C}, & \Psi_{C^{\prime}} \circ \mathrm{E} f^{!} & \simeq \mathrm{E} g^{!} \circ \Psi_{C} .
\end{aligned}
$$

5.2. Enhanced Fourier-Sato transform. Let $M$ be a bordered space, and $U \subset \mathrm{M}$ an open subset. For $\varphi: U \rightarrow \mathbb{R}$ a continuous function, consider the object of $\mathrm{E}_{+}^{\mathrm{b}}\left(\mathrm{I}_{\mathrm{M}}\right)$

$$
\mathrm{E}_{U}^{\varphi}:=\mathrm{Q}_{\mathbf{M}} \mathbf{k}_{\{t+\varphi(x) \geqslant 0\}},
$$

where we write for short

$$
\{t+\varphi(x) \geqslant 0\}=\{(x, t) \in U \times \mathbb{R} ; t+\varphi(x) \geqslant 0\} .
$$

Let $\tau: V \rightarrow N$ be a real vector bundle and $\varpi: V^{*} \rightarrow N$ its dual bundle. Denote by $V_{\infty}$ and $V_{\infty}^{*}$ their bordered compactifications, consider the projections

$$
V_{\infty} \stackrel{p}{\longleftarrow} V_{\infty} \times_{N} V_{\infty}^{*} \stackrel{q}{\longrightarrow} V_{\infty}^{*},
$$

and let $\langle\cdot, \cdot\rangle: V \times_{N} V^{*} \rightarrow \mathbb{R}$ denote the pairing.

Notation 5.2. Let $K \in \mathrm{E}_{+}^{\mathrm{b}}\left(\mathrm{I}_{V_{\infty}}\right)$.

(i) The Fourier-Sato transforms (see [7, §3.7] for the case of sheaves) are defined by

$$
K^{\wedge}:=\Phi_{\mathrm{F}}^{+}(K), \quad K^{\vee}:=\Phi_{7}^{+}(K),
$$

for $\mathrm{F}, \exists \in \mathrm{E}_{+}^{\mathrm{b}}\left(\mathrm{I}_{\mathrm{V}_{\infty} \times_{N} V_{\infty}^{*}}\right)$ given by

$$
\mathrm{F}:=\epsilon^{+}\left(\mathbf{k}_{\{\langle x, y\rangle \leqslant 0\}}\right), \quad \exists:=\epsilon^{+}\left(\mathbf{k}_{\{\langle x, y\rangle \geqslant 0\}} \otimes p^{-1} \omega_{V / N}\right) .
$$

Note that the kernels $\mathrm{F}$ and $\exists$ are $\left(\mathbb{R}_{>0}^{\times}\right)_{\infty}$-bi-conic for the actions $c \cdot(x, y)=(c x, y)$ and $d \cdot(x, y)=(x, d y)$. Hence, the Fourier-Sato transforms take values in $\mathrm{E}_{\left(\mathbb{R}_{>0}^{\times}\right)_{\infty}}^{\mathrm{b}}\left(\mathrm{Ik}_{V_{\infty}^{*}}\right) \cap \mathrm{E}_{+}^{\mathrm{b}}\left(\mathrm{I}_{V_{\infty}^{*}}\right)$.

(ii) The enhanced Fourier-Sato transforms (see [12, §3.1.3] for the case of enhanced sheaves) are defined by

$$
{ }^{\mathrm{L}} K:=\Phi_{\mathrm{L}}^{+}(K), \quad{ }^{\lrcorner} K:=\Phi_{\lrcorner}^{+}(K),
$$

for $\mathrm{L},\lrcorner \in \mathrm{E}_{+}^{\mathrm{b}}\left(\mathrm{I}_{V_{\infty} \times_{N} V_{\infty}^{*}}\right)$ given by

$$
\left.\mathrm{L}:=\mathrm{E}_{V \times N_{N} V^{*}}^{-\langle x, y}, \quad\right\rfloor:=\mathrm{E}_{V \times N}^{\langle x, y\rangle} V^{*} \otimes \pi^{-1} p^{-1} \omega_{V / N} .
$$

Note that the kernels $L$ and $\lrcorner$ are $\left(\mathbb{R}_{>0}^{\times}\right)_{\infty}$-conic for the action $c \cdot(x, y)=\left(c x, c^{-1} y\right)$. Hence the enhanced Fourier-Sato transforms sends $\mathrm{E}_{\left(\mathbb{R}_{>0}^{\times}\right)_{\infty}}^{\mathrm{b}}\left(\mathrm{I} \mathbf{k}_{V_{\infty}}\right)$ to $\mathrm{E}_{\left(\mathbb{R}_{>0}^{\times}\right)_{\infty}}^{\mathrm{b}}\left(\mathrm{I} \mathbf{k}_{V_{\infty}^{*}}\right) \cap \mathrm{E}_{+}^{\mathrm{b}}\left(\mathrm{I} \mathbf{k}_{V_{\infty}^{*}}\right)$. 
It is shown in [12] (see also [9]) that one has

$$
\left.\left.\left(\mathrm{L}_{\circ}^{+}\right\lrcorner^{r}\right)^{\mathrm{prod}} \simeq \mathbf{1}_{V_{\infty}}^{+}, \quad(\lrcorner^{r} \stackrel{+}{\circ} \mathrm{L}\right)^{\mathrm{prod}} \simeq \mathbf{1}_{V_{\infty}^{*}}^{+}
$$

It follows that $\mathrm{L}^{\mathrm{L}}(\cdot)$ and $\lrcorner^{r}(\cdot)$ are quasi-inverse to each other and that, by $(5.3)$

$$
{ }^{\mathrm{L}} K \simeq \Psi_{\lrcorner}^{+}(K), \quad{ }^{\lrcorner} K \simeq \Psi_{\mathrm{L}}^{+}(K) .
$$

Note that one has

$$
e\left(K^{\wedge}\right) \simeq(e(K))^{\wedge}, \quad e\left(K^{\vee}\right) \simeq(e(K))^{\vee},
$$

and the same for $e$ replaced by $\epsilon^{+}$.

The following result was obtained in $[1,9]$ for conic sheaves, and we generalize it to enhanced ind-sheaves.

Proposition 5.3. For $K \in \mathrm{E}_{\left(\mathbb{R}_{>0}^{\times}\right)_{\infty}}^{\mathrm{b}}\left(\mathrm{I}_{V_{\infty}}\right) \cap \mathrm{E}_{+}^{\mathrm{b}}\left(\mathrm{I}_{V_{\infty}}\right)$, one has

$$
{ }^{\mathrm{L}} K \simeq K^{\wedge}, \quad{ }^{\lrcorner} K \simeq K^{\vee}
$$

Proof. We will adapt the proof of [9, Theorem 5.7]. Since the arguments are similar, we will only treat the first isomorphism.

One has

$$
\mathrm{L}=\mathrm{Q} \mathbf{k}_{\{t-\langle x, y\rangle \geqslant 0\}}, \quad \mathrm{F}=\mathrm{Q} \mathbf{k}_{\{t \geqslant 0 \geqslant\langle x, y\rangle\}} .
$$

The inclusion $\{t-\langle x, y\rangle \geqslant 0\} \supset\{t \geqslant 0 \geqslant\langle x, y\rangle\}$ induces a distinguished triangle

$$
\mathrm{Q} \mathbf{k}_{\{t \geqslant\langle x, y\rangle>0\}} \oplus \mathrm{Q} \mathbf{k}_{\{0>t \geqslant\langle x, y\rangle\}} \rightarrow \mathrm{Q} \mathbf{k}_{\{t-\langle x, y\rangle \geqslant 0\}} \rightarrow \mathrm{Q} \mathbf{k}_{\{t \geqslant 0 \geqslant\langle x, y\rangle\}} \stackrel{+1}{\longrightarrow} .
$$

We are thus left to prove

$$
\Phi_{\mathrm{Q} \mathbf{k}_{\{t \geqslant\langle x, y\rangle>0\}}}(K) \simeq 0 \simeq \Phi_{\mathrm{Q} \mathbf{k}_{\{0>t \geqslant\langle x, y\rangle\}}}(K) .
$$

Since the arguments are similar, we will only treat the first isomorphism.

Consider the morphisms

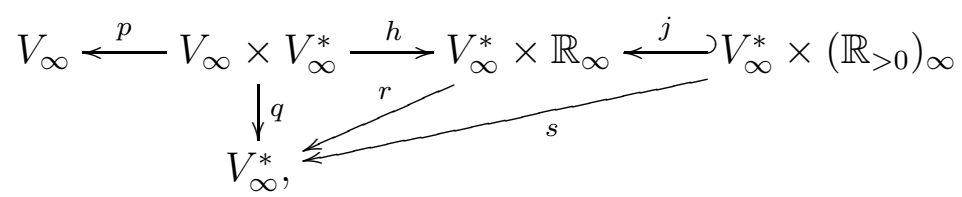


where $h(x, y)=(y,\langle x, y\rangle), j$ is the embedding, and $p, q, r, s$ are the projections. Then, denoting by $\lambda$ the coordinate of $\mathbb{R}_{\infty}$,

$$
\begin{aligned}
& \Phi_{\mathrm{Q} \mathbf{k}_{\{t \geqslant\langle x, y\rangle>0\}}}(K)=\mathrm{E} q_{! !}\left(\mathrm{Q} \mathbf{k}_{\{t \geqslant\langle x, y\rangle>0\}} \stackrel{+}{\otimes} \mathrm{E}^{-1} K\right) \\
& \simeq \mathrm{E} r_{! !} \mathrm{E} h_{! !}\left(\mathrm{E} h^{-1} \mathrm{Q} \mathbf{k}_{\{t \geqslant \lambda>0\}} \stackrel{+}{\otimes} \mathrm{E} p^{-1} K\right) \\
& \simeq \mathrm{Er}_{! !}\left(\mathrm{Q} \mathbf{k}_{\{t \geqslant \lambda>0\}} \stackrel{+}{\otimes} \mathrm{E} h_{! !} \mathrm{E}^{-1} K\right) \\
& \simeq \mathrm{E} r_{! !}\left(\epsilon\left(\mathbf{k}_{\{\lambda>0\}}\right) \stackrel{+}{\otimes} \mathrm{Q} \mathbf{k}_{\{t \geqslant \lambda\}} \stackrel{+}{\otimes} \mathrm{E} h_{! !} \mathrm{E} p^{-1} K\right. \\
& \simeq \mathrm{E} r_{! !} \mathrm{E} j_{! !} \mathrm{E} j^{-1}\left(\mathrm{Q} \mathbf{k}_{\{t \geqslant \lambda\}} \stackrel{+}{\otimes} \mathrm{E} h_{! !} \mathrm{E} p^{-1} K\right) \\
& \simeq \mathrm{E} s_{! !}\left(\mathrm{Q} \mathbf{k}_{\{t \geqslant \lambda\}} \stackrel{+}{\otimes} \mathrm{E} j^{-1} \mathrm{E} h_{! !} \mathrm{E} p^{-1} K\right) \text {. }
\end{aligned}
$$

Since $\mathrm{E} j^{-1} \mathrm{E} h_{! !} \mathrm{E}^{-1} K$ is $\left(\mathbb{R}_{>0}^{\times}\right)_{\infty}$-conic for the action $c \cdot(y, \lambda)=(y, c \lambda)$, we have $\mathrm{E} j^{-1} \mathrm{E}_{! !} \mathrm{E}^{-1} K \simeq \mathrm{Es}^{-1} H$ for some $H \in \mathrm{E}^{\mathrm{b}}\left(\mathrm{Ik}_{V_{\infty}^{*}}\right)$. Hence

$$
\begin{aligned}
\Phi_{\mathrm{Q} \mathbf{k}_{\{t \geqslant\langle x, y\rangle>0\}}}(K) & \simeq \mathrm{E} s_{! !}\left(\mathrm{Q} \mathbf{k}_{\{t \geqslant \lambda\}} \stackrel{+}{\otimes} \mathrm{Es}^{-1} H\right) \\
& \simeq \mathrm{E} s_{! !}\left(\mathrm{Q} \mathbf{k}_{\{t \geqslant \lambda\}}\right) \stackrel{+}{\otimes} H \\
& \underset{(*)}{\simeq} \mathrm{Q}\left(\mathrm{R} s_{\mathbb{R} !} \mathbf{k}_{\{t \geqslant \lambda\}}\right) \stackrel{+}{\otimes} H \simeq 0,
\end{aligned}
$$

where $(*)$ follows since $s$ is semiproper. (Recall that $s_{\mathbb{R}}=s \times \operatorname{id}_{\mathbb{R}}$.)

By Lemma 5.1, we obtain the following analogue of [7, Proposition 3.7.13].

Lemma 5.4. Let $V \rightarrow N$ be a vector bundle and let $f: N^{\prime} \rightarrow N$ be a morphism. Set $V^{\prime}=V \times \times_{N} N^{\prime}$ and $V^{\prime *}=V^{*} \times{ }_{N} N^{\prime}$, and let $g: V^{\prime} \rightarrow V$ and $h: V^{*} \rightarrow V^{*}$ be the induced morphism. Then

(i) For any $K \in \mathrm{E}_{+}^{\mathrm{b}}\left(\mathrm{I}_{V_{\infty}}\right)$, we have

$$
\mathrm{E} h^{-1}\left({ }^{\mathrm{L}} K\right) \simeq{ }^{\mathrm{L}}\left(\mathrm{E} g^{-1} K\right) \text { and } \mathrm{E} h^{!}\left({ }^{\mathrm{L}} K\right) \simeq{ }^{\mathrm{L}}(\mathrm{E} g ! K) \text {. }
$$

(ii) For any $K^{\prime} \in \mathrm{E}_{+}^{\mathrm{b}}\left(\mathrm{I}_{\left(V^{\prime}\right)_{\infty}}\right)$, we have

$$
\mathrm{E} h_{*}\left({ }^{\mathrm{L}} K^{\prime}\right) \simeq{ }^{\mathrm{L}}\left(\mathrm{E} g_{*} K^{\prime}\right) \text { and } \mathrm{E} h_{! !}\left({ }^{\mathrm{L}} K^{\prime}\right) \simeq{ }^{\mathrm{L}}\left(\mathrm{E} g_{! !} K^{\prime}\right) \text {. }
$$

The enhanced Fourier functor satisfies also other functorial properties, as those in Propositions 3.7.14 and 3.7.15 of [7]. The first one was already pointed out in $[9, \S 5.2]$, and the second one easily follows from

$$
\mathrm{E}_{V_{1} \times_{N} V_{1}^{*}}^{-\left\langle x_{1}, y_{1}\right\rangle} \underset{N}{\mathbb{\Delta}} \mathrm{E}_{V_{2} \times_{N} V_{2}^{*}}^{-\left\langle x_{2}, y_{2}\right\rangle} \simeq \mathrm{E}_{\left(V_{1} \times{ }_{N} V_{2}\right) \times_{N}\left(V_{1} \times_{N} V_{2}\right)^{*}}^{-\left\langle\left(x_{1}, x_{2}\right),\left(y_{1}, y_{2}\right)\right\rangle} .
$$

5.3. Enhanced microlocalization. As in $\S 4.3$, let $M$ be a real analytic manifold, and $N \subset M$ a closed submanifold. 
Definition 5.5. For $K \in \mathrm{E}_{+}^{\mathrm{b}}\left(\mathrm{I}_{M}\right)$, we set

$$
\begin{aligned}
& \mathrm{E} \mu_{N}(K):={ }^{\mathrm{L}}\left(\mathrm{E} \nu_{N}(K)\right) \\
& \simeq\left(\mathrm{E} \nu_{N}(K)\right)^{\wedge} \quad \in \mathrm{E}_{\left(\mathbb{R}_{>0}^{\times}\right)_{\infty}}^{\mathrm{b}}\left(\mathrm{Ik}_{\left(T_{N}^{*} M\right)_{\infty}}\right) \cap \mathrm{E}_{+}^{\mathrm{b}}\left(\mathrm{I}_{\left(T_{N}^{*} M\right)_{\infty}}\right), \\
& \mathrm{E} \mu_{N}^{\mathrm{sph}}(K):=\left(\mathrm{E} \mu_{N}(K)\right)^{\mathrm{sph}} \quad \in \mathrm{E}_{+}^{\mathrm{b}}\left(\mathrm{I}_{S_{N}^{*} M}\right),
\end{aligned}
$$

where the isomorphism follows from Proposition 5.3, since $\mathrm{E} \nu_{N}(K)$ is $\left(\mathbb{R}_{>0}^{\times}\right)_{\infty}$-conic. The functor $\mathrm{E} \mu_{N}$ is called enhanced microlocalization along $N$.

Note that one has

$$
e \circ \mu_{N} \simeq \mathrm{E} \mu_{N} \circ e,
$$

and similarly for $e$ replaced by $\epsilon^{+}$.

The enhanced microlocalization functor satisfies the analogous functorial properties as those in Propositions 4.3.4, 4.3.5 and 4.3.6 of [7]. The proofs in loc. cit. immediately extend to the enhanced framework.

\section{Specialization at $\infty$ on Vector Bundles}

On a vector bundle $\tau: V \rightarrow N$, we construct an enhancement of the so-called smash functor from $[2, \S 6.1]$, which is related to "specialization at $\infty "$, and we compute its enhanced Fourier-Sato transform.

6.1. Smash functor. Let $\tau: V \rightarrow N$ be a vector bundle, and consider the morphisms of bordered vector bundles over $N$

$$
V_{\infty} \stackrel{p_{\mathrm{sm}}}{\longleftarrow} V_{\infty} \times\left(\mathbb{R}_{>0}\right)_{\infty} \stackrel{j_{\mathrm{sm}}}{\longrightarrow} V_{\infty} \times \mathbb{R}_{\infty} \stackrel{i_{\mathrm{sm}}}{\longleftarrow} V_{\infty},
$$

where $p_{\mathrm{sm}}(x, s)=s^{-1} x, i_{\mathrm{sm}}(x)=(x, 0)$, and $j_{\mathrm{sm}}$ is the open embedding. In the rest of this section we will write for short $p, i$ and $j$ instead of $p_{\mathrm{sm}}$, $i_{\mathrm{sm}}$ and $j_{\mathrm{sm}}$, respectively, if there is no fear of confusion.

Note that $p, i, j$ are $\left(\mathbb{R}_{>0}^{\times}\right)_{\infty}$-equivariant with respect to the ordinary actions of $\left(\mathbb{R}_{>0}^{\times}\right)_{\infty}$ on $V_{\infty}$ and $\mathbb{R}_{\infty}$, except the trivial action on the leftmost $V_{\infty}$

Definition 6.1. For $K \in \mathrm{E}^{\mathrm{b}}\left(\mathrm{I}_{V_{\infty}}\right)$, set

$$
\mathrm{E} \sigma_{V}(K):=\mathrm{E} i^{-1} \mathrm{E} j_{*} \mathrm{E}^{-1} K \quad \in \mathrm{E}_{\left(\mathbb{R}_{>0}^{\times}\right)_{\infty}}^{\mathrm{b}}\left(\mathrm{I}_{V_{\infty}}\right) .
$$

This is called the enhanced smash functor.

With a proof similar to that of Lemma 4.12, one has

Lemma 6.2. With the above notations, one has

$$
\mathrm{E} \sigma_{V}(K) \simeq \mathrm{E} i ! \mathrm{E} j_{! !} \mathrm{E} p ! K
$$

Lemma 6.3. Let $o: N \rightarrow V$ be the zero section. Then for $K \in \mathrm{E}^{\mathrm{b}}\left(\mathrm{I}_{V_{\infty}}\right)$, one has

$$
\mathrm{E} o^{-1} \mathrm{E} \sigma_{V}(K) \simeq \mathrm{E} \tau_{*} K
$$


Proof. Let $\tilde{\tau}: V_{\infty} \times \mathbb{R}_{\infty} \rightarrow N$ be the projection, and $\tilde{o}: N \rightarrow V_{\infty} \times \mathbb{R}_{\infty}$ the zero section. one has

$$
\begin{aligned}
\mathrm{E} o^{-1} \mathrm{E} \sigma_{V}(K) & \simeq \mathrm{E} o^{-1} \mathrm{E} i^{-1} \mathrm{E} j_{*} \mathrm{E} p^{-1} K \\
& \simeq \mathrm{E} \tilde{o}^{-1} \mathrm{E} j_{*} \mathrm{E} p^{-1} K \\
& \simeq \mathrm{E} \tilde{\tau}_{*} \mathrm{E} j_{*} \mathrm{E} p^{-1} K \\
& \simeq \mathrm{E} \tau_{*} \mathrm{E} p_{*} \mathrm{E} p^{-1} K \\
& \simeq \mathrm{E} \tau_{*} K .
\end{aligned}
$$

Here $(*)$ follows from $\mathrm{E} j_{*} \mathrm{E} p^{-1} K \in \mathrm{E}_{\left(\mathbb{R}_{>0}^{\times}\right)_{\infty}}^{\mathrm{b}}\left(\mathrm{I}_{V_{\infty} \times \mathbb{R}_{\infty}}\right)$.

Consider the vector bundle $W:=\mathbb{R} \times V \rightarrow N$, and let $\dot{W}:=W \backslash(\{0\} \times N)$ be the complement of the zero section. Recall from $\S 4.2$ that the fiberwise sphere compactification ${ }^{2}$ of $\tau$ is $\mathbb{S} V:=\dot{W} / \mathbb{R}_{>0}^{\times}$, We denote by $q: \dot{W} \rightarrow \mathbb{S} V$ the quotient map, and set $[u, x]:=q(u, x)$.

There is a natural decomposition

$$
\mathbb{S} V=V^{+} \sqcup H \sqcup V^{-},
$$

corresponding to $u>0, u=0$ and $u<0$, respectively. Note that the fibers of $H \rightarrow N$ are great spheres of codimension one in the fibers of $\mathbb{S} V \rightarrow N$. Note also that there are natural identifications $\iota^{ \pm}: V \sim V^{ \pm}$, $x \mapsto[ \pm 1, x]$. Set $N^{ \pm}=\iota^{ \pm}(N) \subset V^{ \pm}$, and $N_{0}^{ \pm}=N^{ \pm} \times\{0\} \subset \mathbb{S} V \times \mathbb{R}$.

In order to compute the functor $\mathrm{E} \nu_{H}$, let us describe the normal deformation $\mathbb{S} V_{H}^{\text {nd }}$, and the bordered compactification $\left(\mathbb{S} V_{H}^{\text {nd }}\right)_{\infty}$ of $p_{\text {nd }}: \mathbb{S} V_{H}^{\text {nd }} \rightarrow$ $\mathbb{S} V$.

Lemma 6.4. With notations as above,

(i) one has $\mathbb{S} V_{H}^{\text {nd }} \simeq(\mathbb{S} V \times \mathbb{R}) \backslash\left(N_{0}^{+} \cup N_{0}^{-}\right)$, with $p_{\text {nd }}([\widetilde{u}, \widetilde{x}], s)=[s \widetilde{u}, \widetilde{x}]$ and $s_{\text {nd }}([\widetilde{u}, \widetilde{x}], s)=s$ (see Figure 6.1). The $\mathbb{R}^{\times}$-action on $\mathbb{S} V_{H}^{\text {nd }}$ is given by $c \cdot([\widetilde{u}, \widetilde{x}], s)=\left(([c \widetilde{u}, \widetilde{x}]), c^{-1} s\right)$.

(ii) One has $\left(\mathbb{S} V_{H}^{\text {nd }}\right)_{\infty} \simeq\left((\mathbb{S} V \times \mathbb{R}) \backslash\left(N_{0}^{+} \cup N_{0}^{-}\right), \mathbb{S} V \times \overline{\mathbb{R}}\right)$.

Proof. (i) Set $Z=(u=0) \subset W$. Then $W_{Z}^{\text {nd }}=W \times \mathbb{R}=\mathbb{R} \times V \times \mathbb{R}$ with $p_{\text {nd }}^{W}(\widetilde{u}, \widetilde{x}, s)=(s \widetilde{u}, \widetilde{x})$ and $s_{\text {nd }}^{W}(\widetilde{u}, \widetilde{x}, s)=s$. The $\mathbb{R}^{\times}$-action on $W_{Z}^{\text {nd }}$ is given by $c \cdot(\widetilde{u}, \widetilde{x}, s)=\left(c \widetilde{u}, \widetilde{x}, c^{-1} s\right)$.

One has $\dot{W}_{\dot{Z}}^{\text {nd }}=\left(p_{\text {nd }}^{W}\right)^{-1}(\dot{W})=W_{Z}^{\text {nd }} \backslash((\mathbb{R} \times N \times\{0\}) \cup(\{0\} \times N \times$ $\mathbb{R}))$. Consider the $\mathbb{R}_{>0}^{\times}$-action on $\dot{W}_{\dot{Z}}^{\text {nd }}$ induced by the $\mathbb{R}_{>0}^{\times}$-action on $\dot{W}$, which is given by $c \cdot(\widetilde{u}, \widetilde{x}, s)=(c \widetilde{u}, c \widetilde{x}, s)$. Its quotient is the map $q: \dot{W}_{\dot{Z}}^{\text {nd }} \rightarrow(\mathbb{S} V \times \mathbb{R}) \backslash\left(N_{0}^{+} \cup N_{0}^{-}\right)$, given by $q(\widetilde{u}, \widetilde{x}, s)=([\widetilde{u}, \widetilde{x}], s)$. Setting

\footnotetext{
${ }^{2}$ Here we choose a different compactification from the one in $[2, \S \mathrm{B} .2]$.
} 


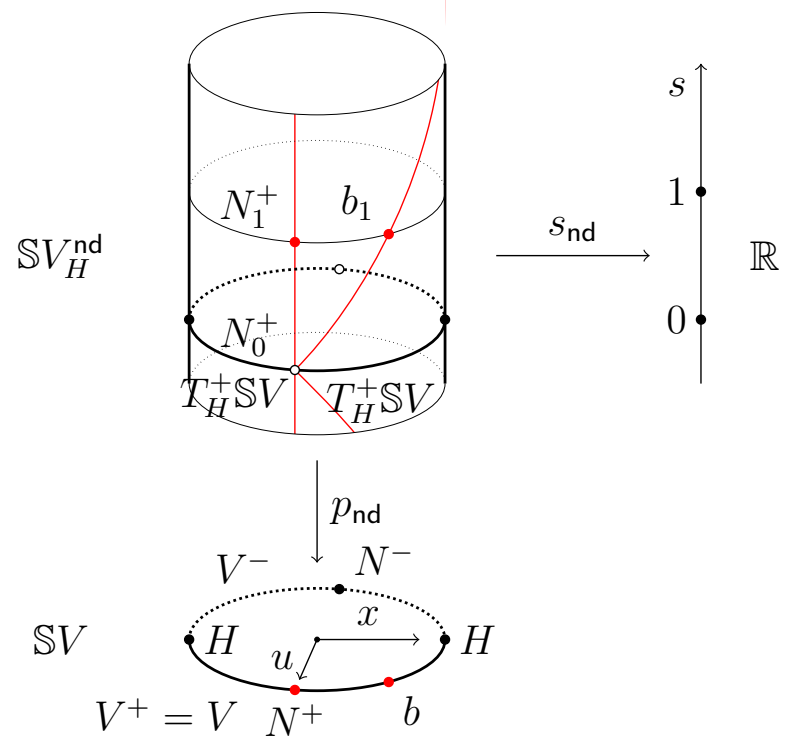

Figure 1. The maps $\mathbb{R} \stackrel{s_{\text {nd }}}{\longleftarrow} \mathbb{S} V_{H}^{\text {nd }} \stackrel{p_{\text {nd }}}{\longrightarrow} \mathbb{S} V$ pictured in the case $\operatorname{dim} V=1$ and $N=\{0\}$. In this case, $H=\{[0,1]\} \sqcup$ $\{[0,-1]\}$. The thick lines in $\mathbb{S} V_{H}^{\text {nd }}$ represent $p_{\text {nd }}^{-1}(H)$. The red lines represent the fibers $p_{\text {nd }}^{-1}\left(N^{+}\right)$and $p_{\text {nd }}^{-1}(b)$, for $b \in$ $V \backslash N$. In the figure, we write $c_{1}=c \times\{1\}$ for $c \in \mathbb{S} V$.

$\tilde{p}([\widetilde{u}, \widetilde{x}], s)=[s \widetilde{u}, \widetilde{x}]$ and $\tilde{s}([\widetilde{u}, \widetilde{x}], s)=s$, there is a commutative diagram with cartesian square

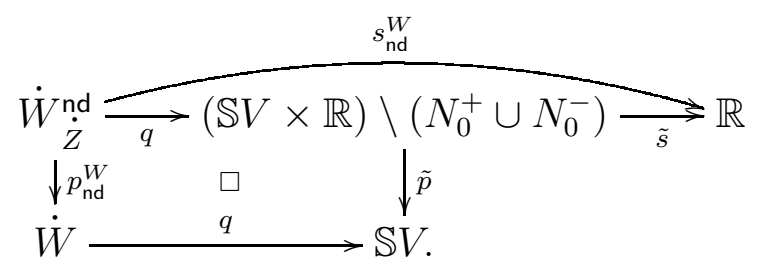

Since the quotient maps $q$ are principal $\mathbb{R}_{>0}^{\times}$-bundles, it follows that $\mathbb{S} V_{H}^{\text {nd }}=(\mathbb{S} V \times \mathbb{R}) \backslash\left(N_{0}^{+} \cup N_{0}^{-}\right), p_{\text {nd }}=\tilde{p}$ and $s_{\text {nd }}=\tilde{s}$.

(ii) follows by uniqueness of bordered compactifications.

Denote by $T_{H}^{+} \mathbb{S} V \subset \dot{T}_{H} \mathbb{S} V$ the normal vectors pointing to $V^{+}$. Since

$$
T_{H} \mathbb{S} V=s_{\text {nd }}^{-1}(0)=\left(\dot{V}^{+} \sqcup H \sqcup \dot{V}^{-}\right) \times\{0\},
$$

this gives a natural identification $T_{H}^{+} \mathbb{S} V=\dot{V}^{+}$. We will also use the identification $V=V^{+}$given by $\iota^{+}$. Note that the $\mathbb{R}_{>0^{-}}^{\times}$action on $\dot{V}^{+} \subset$ $T_{H} \mathbb{S} V$ induced by the one on $T_{H} \mathbb{S} V$ is given by $c \cdot \iota^{+}(x)=\iota^{+}\left(c^{-1} x\right)$.

By Lemma 4.9, with the above identifications, $\mathrm{E} \nu_{H}$ induces a functor

$$
\mathrm{E} \nu_{H \mid \dot{V}}: \mathrm{E}^{\mathrm{b}}\left(\mathrm{Ik}_{\dot{V}_{\infty}}\right) \rightarrow \mathrm{E}^{\mathrm{b}}\left(\mathrm{Ik}_{\dot{V}_{\infty}}\right)
$$


Similarly, E $\sigma_{V}$ induces a functor (see (6.2) below)

$$
\mathrm{E} \sigma_{V \mid \dot{V}}: \mathrm{E}^{\mathrm{b}}\left(\mathrm{Ik}_{\dot{V}_{\infty}}\right) \rightarrow \mathrm{E}^{\mathrm{b}}\left(\mathrm{Ik}_{\dot{V}_{\infty}}\right)
$$

Lemma 6.5. With the above notations, one has

$$
\mathrm{E} \nu_{H \mid \dot{V}} \simeq \mathrm{E} \sigma_{V \mid \dot{V}}
$$

Proof. Consider the diagram with cartesian squares

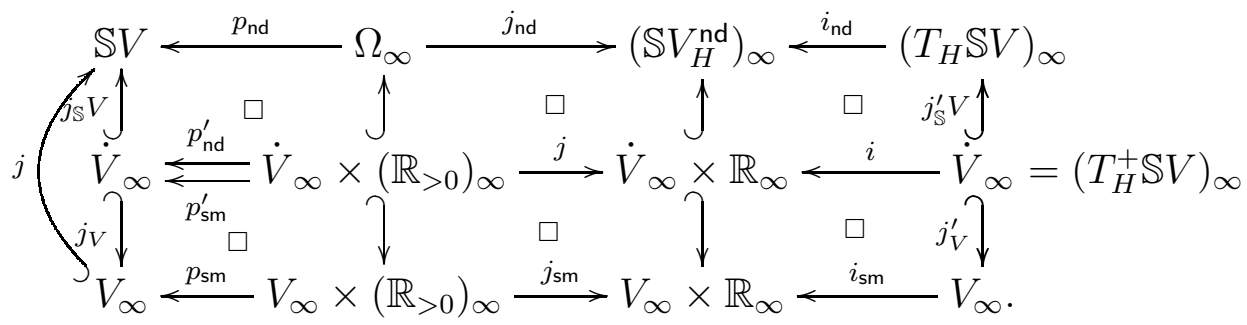

Here, $p_{\mathrm{sm}}^{\prime}$ and $p_{\text {nd }}^{\prime}$ are induced by $p_{\mathrm{sm}}$ and $p_{\text {nd }}$, respectively.

Since

$$
\begin{aligned}
j_{\mathbb{S}} V\left(p_{\text {nd }}^{\prime}(x, s)\right) & =p_{\text {nd }}([1, x], s)=[s, x]=\left[1, s^{-1} x\right], \\
j\left(j_{V}\left(p_{\mathrm{sm}}^{\prime}(x, s)\right)\right) & =j\left(p_{\mathrm{sm}}(x, s)\right)=j\left(s^{-1} x\right)=\left[1, s^{-1} x\right],
\end{aligned}
$$

one has in fact $p_{\text {nd }}^{\prime}=p_{\mathrm{sm}}^{\prime}$.

By (4.6), one has

$$
\mathrm{E} \nu_{H \mid \dot{V}} \simeq \mathrm{E} i^{-1} \circ \mathrm{E} j_{*} \circ \mathrm{E}\left(p_{\text {nd }}^{\prime}\right)^{-1} .
$$

Similar arguments give

$$
\mathrm{E} \sigma_{V \mid \dot{V}} \simeq \mathrm{E} i^{-1} \circ \mathrm{E} j_{*} \circ \mathrm{E}\left(p_{\mathrm{sm}}^{\prime}\right)^{-1} .
$$

6.2. Smash functor and microlocalization. As in the previous section, let $\tau: V \rightarrow N$ be a vector bundle. Denote by $o: N \rightarrow V$ the embedding of the zero section. Consider the natural identifications

$$
N=o(N) \subset V, \quad T_{N}^{*} V=V^{*} .
$$

There is the following relation between the smash functor and the FourierSato transform

Proposition 6.6. For $K \in \mathrm{E}_{+}^{\mathrm{b}}\left(\mathrm{I}_{V_{\infty}}\right)$ there is an isomorphism in $\mathrm{E}_{+}^{\mathrm{b}}\left(\mathrm{I}_{V_{\infty}^{*}}\right)$

$$
\mathrm{E} \mu_{N}(K) \simeq \mathrm{E} \sigma_{V^{*}}\left({ }^{\mathrm{L}} K\right) .
$$

In other words, one has $\mathrm{L}^{\mathrm{L}}(\cdot) \circ \mathrm{E} \nu_{N} \simeq \mathrm{E} \sigma_{V^{*}} \circ \mathrm{L}(\cdot)$. 
Proof. Consider the following diagram with cartesian squares.

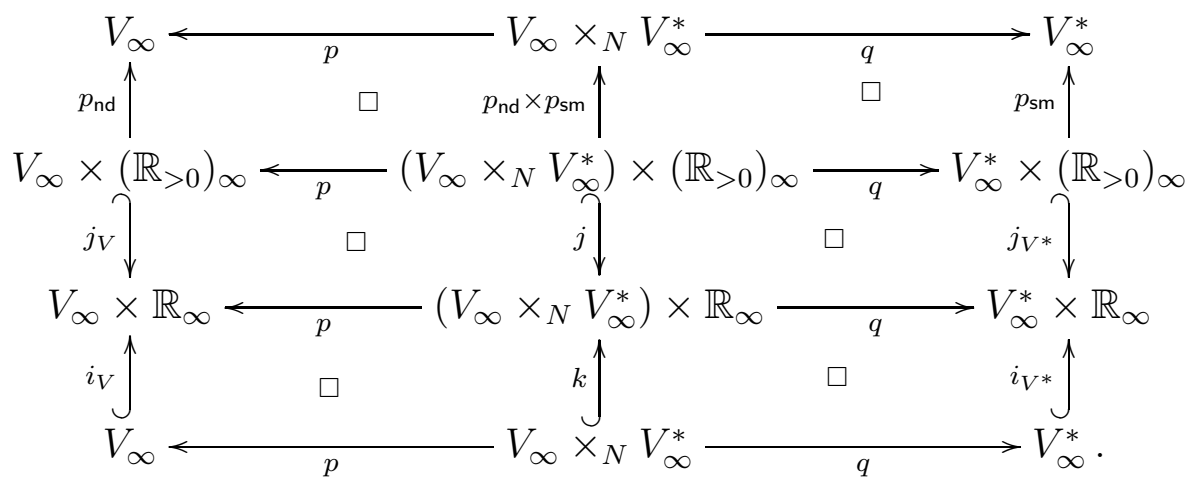

We have to prove

$$
{ }^{\mathrm{L}}\left(\mathrm{E} i_{V}^{-1} \mathrm{E} j_{V *} \mathrm{E} p_{\text {nd }}^{-1} K\right) \simeq \mathrm{E} i_{V^{*}}^{-1} \mathrm{E} j_{V^{*}{ }_{*}} \mathrm{E} p_{\text {sm }}^{-1}\left({ }^{\mathrm{L}} K\right) .
$$

Consider the left and right columns as morphisms of vector bundles over $N, N \times \mathbb{R}_{>0}, N \times \mathbb{R}$ and $N$, respectively. Then the left column is the dual of the right column, and the middle column is the vector bundle product of the left and the right columns. Moreover, since $\left(p_{\mathrm{rb}} \times\right.$ $\left.p_{\mathrm{sm}}\right)(x, y, s)=\left(s x, s^{-1} y\right)$ and $\left\langle s x, s^{-1} y\right\rangle=\langle x, y\rangle$, the maps from the second row to the top row is compatible with the coupling of the left columns and the right columns.

Therefore (6.3) follows from Lemma 5.4.

\section{REFERENCES}

[1] A. D'Agnolo, On the Laplace transform for tempered holomorphic functions, Int. Math. Res. Not. 2014 no. 16 (2014), 4587-4623.

[2] A. D'Agnolo, M. Hien, G. Morando, C. Sabbah, Topological computations of some Stokes phenomena, Ann. Inst. Fourier, to appear, preprint arXiv:1705.07610v2 (2018), $51 \mathrm{pp}$.

[3] A. D'Agnolo and M. Kashiwara, Riemann-Hilbert correspondence for holonomic D-modules, Publ. Math. Inst. Hautes Études Sci. 123 (2016), no. 1, 69-197.

[4] _ Enhanced perversities, J. Reine Angew. Math. (Crelle's Journal) 751 (2019), 185-241.

[5] S. Guillermou and P. Schapira, Microlocal theory of sheaves and Tamarkin's non displaceability theorem, in: Homological Mirror Symmetry and Tropical Geometry, Lecture Notes of the Unione Matematica Italiana 15, Springer, Berlin (2014), $43-85$.

[6] M. Kashiwara, Riemann-Hilbert correspondence for irregular holonomic $\mathcal{D}$ modules, Jpn. J. Math. 11 (2016), no. 1, 113-149.

[7] M. Kashiwara and P. Schapira, Sheaves on manifolds, Grundlehren der Mathematischen Wissenschaften 292, Springer, Berlin (1990), $\mathrm{x}+512$ pp.

[8] _ Ind-sheaves, Astérisque 271 (2001), 136 pp.

[9] _ Irregular holonomic kernels and Laplace transform, Selecta Math. 22 (2016), no. 1, 55-109.

[10] _ Regular and irregular holonomic D-modules, London Mathematical Society Lecture Note Series 433, Cambridge University Press, Cambridge (2016), vi $+111 \mathrm{pp}$. 
[11] M. Kashiwara, P. Schapira, F. Ivorra and I. Waschkies, Microlocalization of indsheaves, in: Studies in Lie theory, 171-221, Progr. Math. 243, Birkhäuser (2006).

[12] D. Tamarkin, Microlocal condition for non-displaceability, in: Algebraic and Analytic Microlocal Analysis, Springer Proc. in Math. \& Stat. 269 (2018), 99-223.

(Andrea D'Agnolo) Dipartimento di Matematica, Università di Padova, via Trieste 63, 35121 Padova, Italy

E-mail address: dagnolo@math.unipd.it

(Masaki Kashiwara) Research Institute for Mathematical Sciences, Kyoto University, Kyoto 606-8502, Japan \& Korea Institute for Advanced Study, SeOul 02455, Korea

E-mail address: masaki@kurims.kyoto-u.ac.jp 